\title{
Prospects for Galactic transient sources detection with the Cherenkov Telescope Array
}

\author{
Alicia López-Oramas, ${ }^{a, b, *}$ A. Bulgarelli, ${ }^{c}$ S. Chaty, ${ }^{d}$ M. Chernyakova, ${ }^{e}$ R. Gnatyk, ${ }^{f}$ B. \\ Hnatyk, ${ }^{f}$ D. Kantzas, ${ }^{g}$ S. Markoff, ${ }^{g}$ S. McKeague, ${ }^{e}$ S. Mereghetti, ${ }^{h}$ E. Mestre, ${ }^{i}$ A. di \\ Piano, ${ }^{c}$ P. Romano, ${ }^{j}$ I. Sadeh, ${ }^{k}$ O. Sergijenko, ${ }^{f}$ L. Sidoli, ${ }^{h}$ A. Spolon, ${ }^{l}$ E. de Ona \\ Wilhelmi, ${ }^{k}$ G. Piano ${ }^{m}$ and L. Zampieri ${ }^{n}$ on behalf of the CTA Consortium \\ ${ }^{a}$ Inst. de Astrofísica de Canarias, La Laguna, Spain \\ ${ }^{b}$ Universidad de La Laguna, Dpto. Astrofísica, La Laguna, Tenerife, Spain \\ ${ }^{c}$ INAF-OAS Bologna, Italy \\ ${ }^{d}$ University of Paris and CEA Paris-Saclay, France \\ ${ }^{e}$ School of Physical Sciences and CfAR, Dublin City University, Ireland \\ ${ }^{f}$ Taras Shevchenko National University of Kyiv, Ukraine \\ ${ }^{g}$ API/GRAPPA, University of Amsterdam, the Netherlands \\ ${ }^{h}$ INAF-IASF Milano, Italy \\ ${ }^{i} I C E-C S I C$, Barcelona, Spain \\ ${ }^{j}$ INAF-Osservatorio Astronomico di Brera, Milano, Italy \\ ${ }^{k}$ DESY-Zeuthen, Zeuthen, Germany \\ ${ }^{l}$ Università di Padova and INFN, Padova, Italy \\ ${ }^{m}$ INAF-IAPS Roma, Italy \\ ${ }^{n}$ INAF-Astronomical Observatory of Padova, Italy \\ E-mail: alicia.lopez@iac.es
}

Several types of Galactic sources, like magnetars, microquasars, novae or pulsar wind nebulae flares, display transient emission in the X-ray band. Some of these sources have also shown emission at $\mathrm{MeV}-\mathrm{GeV}$ energies. However, none of these Galactic transients have ever been detected in the very-high-energy (VHE; E >100 GeV) regime by any Imaging Air Cherenkov Telescope (IACT). The Galactic Transient task force is a part of the Transient Working group of the Cherenkov Telescope Array (CTA) Consortium. The task force investigates the prospects of detecting the VHE counterpart of such sources, as well as their study following Target of Opportunity (ToO) observations. In this contribution, we will show some of the results of exploring the capabilities of CTA to detect and observe Galactic transients; we assume different array configurations and observing strategies.

$37^{\text {th }}$ International Cosmic Ray Conference (ICRC 2021)

July 12 th - 23rd, 2021

Online - Berlin, Germany

${ }^{*}$ Presenter 


\section{Introduction}

Many different types of sources in the Galaxy exhibit transient signals. Such emission can occur due to accretion/ejection processes, such as jets interacting with the interstellar medium (ISM), strong winds and/or outflows. In these scenarios, particles may be accelerated up to relativistic energies, leading to the production of high-energy (HE, E >100 MeV) and likely very-high-energy (VHE, E $>100 \mathrm{GeV}$ ) radiation, via leptonic or hadronic processes. While many Galactic sources show variable periodic signals, we are interested in those that display irregular unpredictable emission at different wavelengths.

Several types of Galactic transients exhibiting HE radiation have been detected in the past years by satellites such as Fermi-LAT and AGILE. In 2011, AGILE discovered enhanced MeV emission from the Crab Nebula pulsar wind nebula (PWN) [1], confirmed by Fermi-LAT[2]. This indicates that this source, considered the VHE standard candle, is actually variable at lower energies. Recently, a Galactic magnetar (SGR $1935+2157$ ) has been associated with a Fast Radio Burst (FRB) for the first time [3]. This event was not detected in the gamma-ray regime. However, the Fermi-LAT has recently discovered $\mathrm{GeV}$ emission from an extragalactic magnetar, located in the Sculptor galaxy, which occurred during a giant flare [4]. Microquasars, which are binary systems hosting compact objects -either neutron stars (NS) or black holes (BH)- accreting from a companion star, can lead to the formation of a jet. Some microquasars have also been detected in the HE regime [5-7]. Additionally, novae, which are explosions associated to a white dwarf in a close binary system, have been detected in the $\mathrm{MeV}$ regime [8]. Finally, transitional millisecond pulsars (tMSPs) are pulsars in a binary system, which change from an accretion to a radio loud phase; such tMSPs have also been detected in the $\mathrm{MeV}$ energy range [9].

The current generation of Imaging Air Cherenkov Telescopes (IACTs) are H.E.S.S. [10], MAGIC [11], and VERITAS [12]. They have successfully discovered more than 200 VHE gammaray sources, both of galactic and extragalactic origin. Highlights in the case of Galactic sources include the discovery of gamma-ray binaries with variable emission [13-17], pulsations in the $\mathrm{GeV}-\mathrm{TeV}$ domain in the emission of pulsars, such as the Crab [18], Vela [19] or Geminga [19] or emission from the Galactic centre, revealing it as the first PeVatron (source of cosmic rays with PeV energies) in the Galaxy [20], among others. IACTs have been aiming at detecting other types of transient emission, such as the aformentioned sources, without success [21-24].

The Cherenkov Telescope Array (CTA) will be the next-generation ground-based gammaray observatory [25]. It will be the premier facility for VHE, multi-messenger, and transients astrophysics in the next decade. CTA will comprise two observatories, one in the Northern (Observatorio Roque de los Muchachos, La Palma) and one in the Southern hemisphere (Paranal, Chile). CTA will be able to perform unprecedented observations of VHE transient sources, covering an energy range from $20 \mathrm{GeV}$ to more than $100 \mathrm{TeV}$. The larger effective area of CTA, compared to the current generation of IACTs, will result in high sensitivity ${ }^{1}$ at short timescales (see Fig.1) [26]. This will enable unique studies of the multi-messenger and transient sky [27], which is one of Key Science Projects of the observatory [25]. It is correspondingly necessary to carefully understand the capabilities of CTA to detect such sources (see Carosi, ICRC2021, id.833). In many cases, CTA observations will be based on external triggers from various monitoring instruments (X-ray or HE

\footnotetext{
${ }^{1}$ CTA sensitivity: https://www.cta-observatory.org/science/cta-performance/
} 


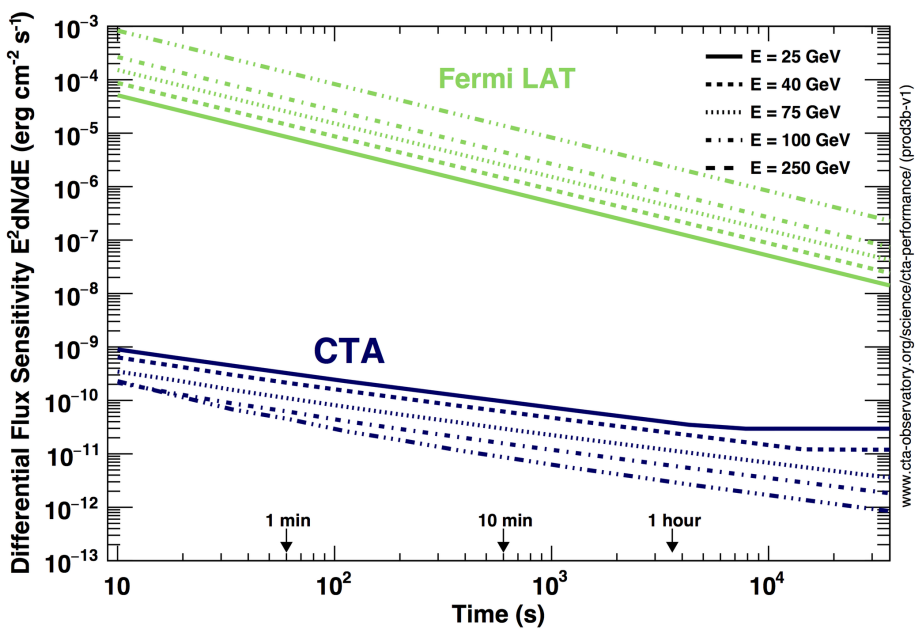

Figure 1: The differential flux sensitivities of CTA and of Fermi-LAT for different energies as a function of observation time.

satellites). Serendipitous discoveries can also take place, i.e., as part of the nominal observation of the Galactic plane survey (GPS). The Science Alert Generation, which is a real-time very-short timescale (from 1 to 100 seconds) analysis will play a key role in the follow-up of external triggers and in the serendipitous discovery of transient events (see di Piano, ICRC2021, id.156).

While certain objects show persistent emission and/or periodically emit variable radiation, in this contribution we will mainly focus on those emitters on those emitters which display irregular and unpredictable transient emission at different wavelengths. We will summarize the work of the Galactic transients task force of the CTA Consortium, which is focused in understanding the capabilities of CTA for detecting transient sources of Galactic origin. The results shown in this contribution will be discussed in more detail in an upcoming CTA Consortium paper.

\section{Sensitivity in the Galactic plane}

It is important to understand the performance of the CTA Northern and Southern arrays, especially in the Galactic plane, where many $\mathrm{TeV}$ sources are located. The sensitivity of the Southern array is illustrated in Fig. 2 for different perspective source locations, and is defined as the minimal flux of a source, such that the source is detectable at $5 \sigma$ significance within a given energy range. We define the variable $S$, which stands for the sensitivity multiplied by the energy squared. For the current example, we estimate the performance of CTA for short observation intervals of 60 seconds, within the $100-200 \mathrm{GeV}$ energy range.

As it can be inferred from the figure, upward fluctuations of the sensitivity (worse performance of CTA), are correlated with the simulated Galactic emission. The flux of the expected steady Galactic foreground in the chosen energy range is mostly below the level of a few $10^{-11} \mathrm{erg} \mathrm{cm}^{2} \mathrm{~s}^{-1}$. This is of the same order as the nominal sensitivity of the observatory in the absence of foregrounds. Correspondingly, the overall degradation in sensitivity for detection of new sources is not significant; 
at worst, it amounts to a relative increase of the flux threshold of 5-10\%, and only when coinciding with strong Galactic emitters.

As part of our upcoming publication, we will explore the performance along the Galactic plane for different energy ranges and observation times. Given the assumed properties of known Galactic sources, the performance is not expected to significantly diverge from the nominal capabilities of CTA, which are shown in Fig. 1.
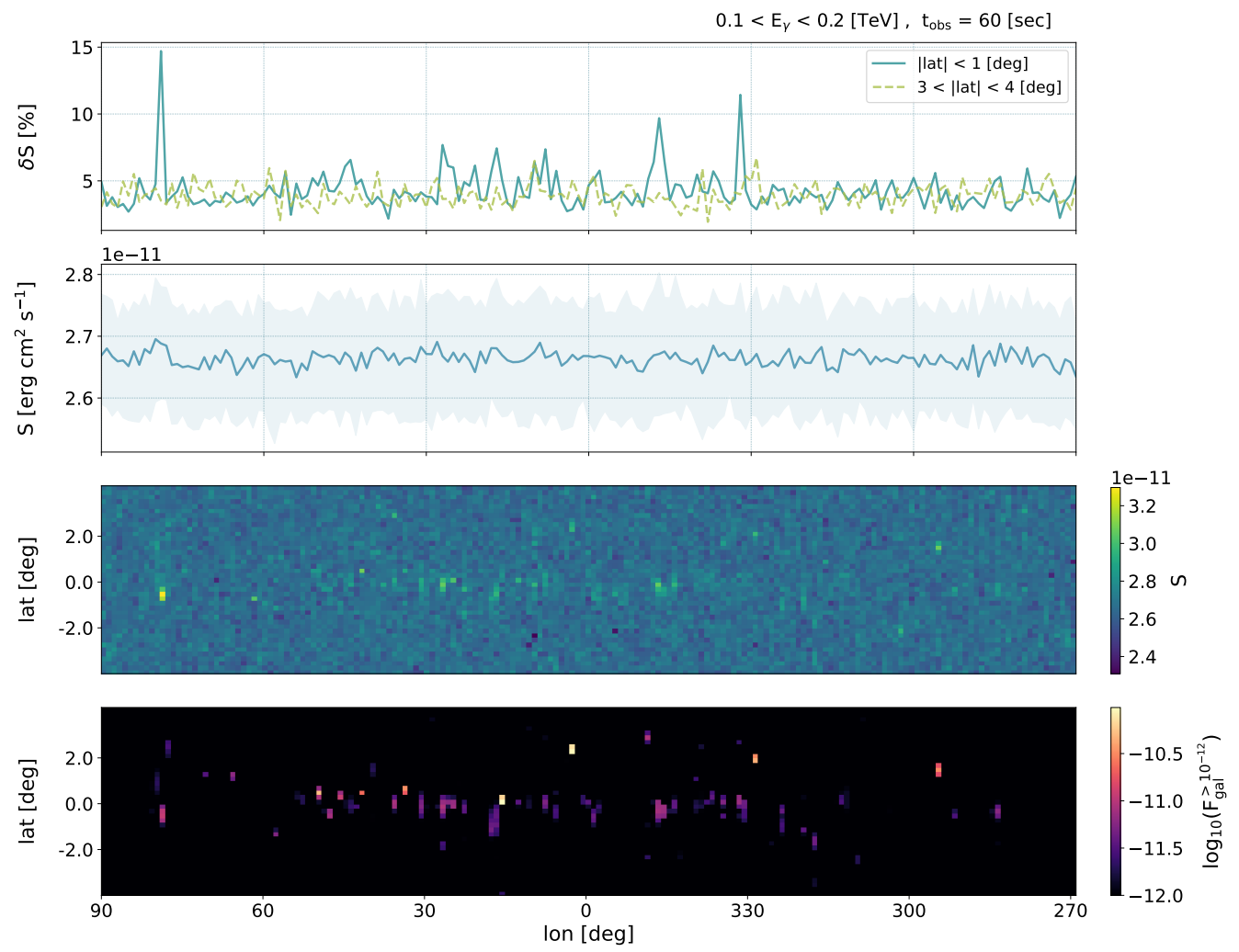

Figure 2: Flux sensitivity $(S)$ of CTA-S within 100-200 GeV for 60 s observation intervals, considering different perspective source locations along the Galactic plane. The bottom panel shows a simulation of $F_{\text {gal }}^{>10^{-12}}$, the Galactic emission above a threshold of $10^{-12} \mathrm{erg} \mathrm{cm}^{2} \mathrm{~s}^{-1}$. The emission is integrated within $0.25^{\circ}$ radial regions around each position, corresponding to different Galactic longitudes, lon, and latitudes, lat. The next panel above shows the corresponding CTA sensitivity. In the third panel we present the median of $S$ for different longitudes within the range, $-4<$ lat $<4$ deg, where the shaded uncertainty region represents the $1 \sigma$ variance of $S$. Finally, the top panel shows the relative $1 \sigma$ variance, $\delta S$, derived for two ranges in latitude, as indicated. The variance away from the Galactic Plane $(3<\mid$ lat $\mid<4$ deg $)$ represents the intrinsic statistical uncertainty of the sensitivity calculation. The variance in the inner Galactic region ( $\mid$ lat $\mid<1 \mathrm{deg}$ ) includes the intrinsic uncertainty, as well as the additional effect of the steady Galactic foregrounds, which are concentrated in this region.

\section{Detecting Galactic transients with CTA}

We have tested the capabilities of CTA to detect transient VHE emission for different kinds of Galactic sources. We have assumed different array configurations, namely the full CTA Northern 
(CTA-N), and CTA Southern (CTA-S) arrays, as well as different sub-arrays of CTA telescopes, different observing strategies, etc. Below we illustrate our findings with three source examples, microquasars, flaring PWNe and tMSPs, which are sources that are known to show MeV emission.

\subsection{Microquasars}

Microquasars are binary systems composed of a compact object (BH or NS) which accretes matter from a companion star. Depending on the mass of the companion, they can be divided into high-mass or low-mass systems. Microquasars normally present an accretion disk, and can produce collimated jets of plasma. These jets are normally active during the so-called hard state.

We have studied three microquasars located in the Cygnus region: the two high-mass systems Cyg X-1 and Cyg X-3 and low-mass binary V404 Cyg. Both Cyg X-1 and Cyg X-3 are sources of HE gamma rays [5-7], although the nature of their emission mechanisms (hadronic or leptonic) is still uncertain. Correspondingly, the emission may be attributed to a jet interacting with the surrounding interstellar material (ISM), or from the coronal region of the accretion flow. The latter scenario is quite interesting, indicating that microquasars are potential accelerators of cosmic rays via magnetic reconnection [28].

Searches for both transient and persistent VHE emission from microquasars have not resulted in detections, either from the binaries themselves, or from jet-ISM interactions [29, 30]. Only, a hint of transient emission from Cyg X-1 was observed by the MAGIC telescopes in 2006, over an 80-minute observation interval [31]. The low-mass binary V404 Cyg displayed a major flaring episode in X-rays in June 2015, after 26 years in quiescence; unfortunately, only $4 \sigma$ evidence was observed by Fermi-LAT [32], falling short of a correlated detection. No VHE emission was observed either [33].

We have tested different scenarios in which we would expect transient and persistent emission from any of these binary systems. CTA will not be able to detect a flare from V404 Cyg. However, our simulations indicate that CTA will detect transient emission in both Cyg X-1 and Cyg X-3, where we wish to highlight the expected detection of Cyg X-1 in only 0.5 h, as shown in Fig.3.

For a small number of microquasars, the jets are persistent, such as for SS433. This microquasar has been detected in the $\mathrm{MeV}$ range by Fermi-LAT [34]; it is the only one also detected in the TeV range, as reported by HAWC [17]. The observed extended $\mathrm{TeV}$ emission is due to the interaction between the jet and the surrounding nebula (the so-called lobes), while the central binary remains undetected. To date, this source has not been detected by an IACT [35], which would have helped to fill in the as-yet unexplored GeV-TeV energy range between Fermi-LAT and HAWC. Our simulations indicate that both the CTA-N and CTA-S arrays will be able to detect the central binary SS433 and its lobes with high significance. CTA will thus provide crucial insight into the emission models of microquasars, and advance our understanding of jet formation.

\subsection{Flares from PWNe}

The discovery of MeV flares from the Crab Nebula $[1,2]$ revealed that PWNe can also display transient variable emission, even if most of these systems are detected as steady sources. These flares, however, have never been detected in the GeV-TeV regime. The CTA observatory will advance our understanding of the origin of the Crab Nebula flares. The low-energy threshold of 
CTA will allow sampling of the Fermi-LAT spectral shape. Complementary observations in the $\mathrm{TeV}$ regime will be used to explore a possible inverse Compton (IC) component of the emission, which might arise via the off-scattering of the $\mathrm{MeV}$ flares.

We have tested how the Northern CTA array will detect such flaring episodes, using two array configurations. The first is the full CTA array (CTA-N), including four Large Size Telescopes (LSTs) and 15 Medium Size Telescopes (MSTs). The second configuration is a partial sub-array of telescopes, composed exclusively of four LSTs (CTA-N LSTs), which dominate the low-energy sensitivity range of the observatory. Our conclusion is that CTA will be able to detect these flares in less than $5 \mathrm{~h}$, even if only the reduced 4-LST sub-array is available. The results for detecting flaring episodes with different flux levels is show in Fig.3.
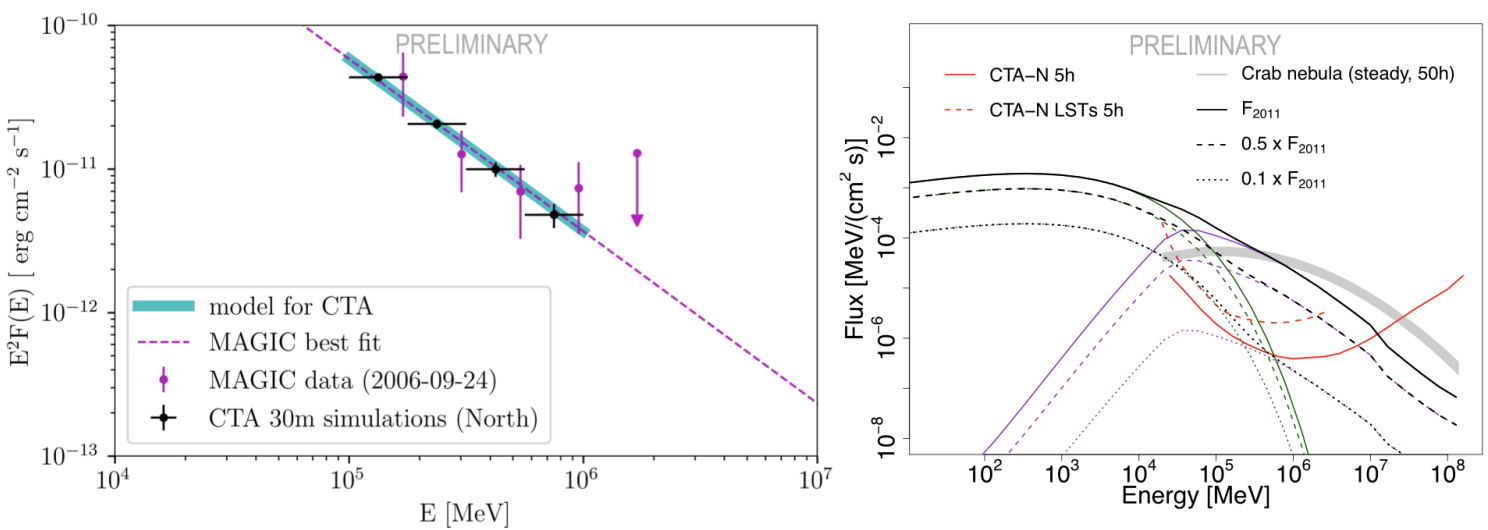

Figure 3: Left: Spectral energy distribution of Cyg X-1 during a flaring episode similar to that reported in [31] (magenta points). CTA will detect this binary in 30 minutes of observation (black points; model in cyan). Right: simulation of different flaring models (black lines), result of the synchrotron (green) and IC (purple) contributions. The red lines correspond to the sensitivities of CTA-North and the 4 Large Size Telescopes (LSTs) of CTA-North (for $5 \mathrm{~h}$ ). The steady Crab spectrum is plotted for comparison (gray shaded area). Figures extracted from the CTA Consortium paper on Galactic transients (in prep.)

\subsection{Transitional millisecond pulsars (tMSPs)}

tMPS are binaries composed of a low-mass star and a pulsar with a millisecond-duration period. These systems change from an accretion-powered phase to a radio loud phase. Three tMSPs have been detected at HE by Fermi-LAT during the accretion-powered phase [9]. Transitions between the two states can occur on timescales from days to weeks, producing variability in the whole electromagnetic spectrum. We have tested whether CTA could detect emission from two systems, PSR J1023+0038 and XSS J1227-48538, which are detected with Fermi-LAT in the 0.1-10 GeV energy range during the accretion phase. We show that the full CTA array will be able to detect persistent emission from these sources by performing long integration-time observations $(>50 \mathrm{~h})$.

\section{Summary}

CTA will perform real-time TeV studies of the variable Galactic sky. Its unique sensitivity to short-timescale events and its low energy threshold make this observatory a powerful and efficient 
instrument to detect and discover new transient sources. The observational strategy of CTA includes both follow-up of externally triggered events, and serendipitous discoveries, taking place e.g., while conducting a Galactic plane Survey. CTA will be able to detect microquasars such as Cyg X-1, Cyg X-3 and SS433 in the GeV-TeV domain for the first time. It will also be able to probe the flaring episodes of PWNe (namely the Crab Nebula) at VHE. CTA will detect for the first time the VHE component of tMSPs, by performing dedicated long-time observations ( $>50 \mathrm{~h})$ during the accretion state.

The unique capabilities of CTA will also likely result in the detection of other large variety of Galactic transients, such as novae, flares from known gamma-ray/X-ray binaries and magnetars. Serendipitous discoveries are also possible. CTA will play a key role in identifying the nature of new transients by performing follow-up observations of wide field-of-view instruments. CTA will help to reveal their most energetic counterpart, and to unveil the acceleration mechanisms at work. The real-time analysis Science Alert Generation will play a key role in the follow-up and observation strategies of externally triggered events and also in the serendipitous discovery of transient events.

Acknowledgements: This research made use of ctools, a community-developed analysis package for Imaging Air Cherenkov Telescope data. ctools is based on GammaLib, a community-developed toolbox for the scientific analysis of astronomical gamma-ray data.

\section{References}

[1] M. Tavani, A. Bulgarelli, V. Vittorini, et al. (AGILE), Science 331, 736 (2011), 1101.2311.

[2] A. A. Abdo et al. (Fermi-LAT), Science 331, 739 (2011), 1011. 3855.

[3] B. C. Andersen et al. (CHIME/FRB), Nature 587, 54 (2020), 2005.10324.

[4] M. Ajello et al. (Fermi-LAT), Nature Astronomy 5, 385-39 (2021).

[5] A. A. Abdo et al. (Fermi-LAT), Science 326, 1512 (2009).

[6] S. Sabatini, M. Tavani, E. Striani, et al., Astrophysical Journal Letters 712, L10 (2010), 1002 . 4967.

[7] R. Zanin et al., Astronomy and Astrophysics 596, A55 (2016), 1605.05914.

[8] A. A. Abdo et al. (Fermi-LAT), Science 329, 817 (2010), 1008. 3912.

[9] P. S. Ray et al. (Fermi Pulsar Search Consortium) (2012), 1205 . 3089.

[10] F. Aharonian et al. (H.E.S.S.), Astronomy and Astrophysics 457, 899 (2006), astro-ph/0607333.

[11] J. Aleksić et al. (MAGIC), Astroparticle Physics 72, 76 (2016), 1409. 5594.

[12] J. Holder (VERITAS), in Proceedings SciNeGHE 2006 (2007), pp. 69-76, astro-ph/0611598.

[13] F. Aharonian, A. G. Akhperjanian, K. M. Aye, et al. (H.E.S.S.), Astronomy and Astrophysics 442, 1 (2005), astro-ph/0506280.

[14] F. Aharonian, A. G. Akhperjanian, K. M. Aye, et al. (H.E.S.S.), Science 309, 746 (2005), astro-ph/ 0508298. 
[15] J. Albert, E. Aliu, H. Anderhub, et al. (MAGIC), Science 312, 1771 (2006), astro-ph/0605549.

[16] F. A. Aharonian, A. G. Akhperjanian, A. R. Bazer-Bachi, et al. (H.E.S.S.), Astronomy and Astrophysics 469, L1 (2007), 0704. 0171.

[17] A. U. Abeysekara, W. Benbow, R. Bird, et al. (VERITAS and MAGIC), Astrophysical Journal Letters 867, L19 (2018), 1810.05271.

[18] J. Albert, E. Aliu, H. Anderhub, et al. (MAGIC), The Astrophysical Journal 674, 1037 (2008), 0705. 3244.

[19] H. Abdalla, F. Aharonian, F. Ait Benkhali, et al. (H.E.S.S.), Astronomy and Astrophysics 620, A66 (2018), 1807.01302.

[20] A. Abramowski, F. Aharonian, F. A. Benkhali, et al. (H.E.S.S.), 531, 476 (2016), 1603.07730.

[21] J. Aleksić, L. A. Antonelli, P. Antoranz, et al. (MAGIC), Astronomy and Astrophysics 549, A23 (2013), 1211.1173.

[22] A. López-Oramas, O. Blanch, E. de Oña Wilhelmi, et al., IJMP D 27, 1844010 (2018), 1807 . 09509.

[23] M. L. Ahnen, S. Ansoldi, L. A. Antonelli, et al. (MAGIC), Astronomy and Astrophysics 582, A67 (2015), 1508.04902.

[24] E. Aliu, S. Archambault, T. Arlen, et al. (VERITAS), The Astrophysical Journal 754, 77 (2012), 1205.5287.

[25] B. S. Acharya et al. (CTA Consortium) (WSP, 2018), ISBN 9789813270084, 1709. 07997.

[26] V. Fioretti, D. Ribeiro, T. B. Humensky, et al., in 36th International Cosmic Ray Conference (ICRC2019) (2019), vol. 36 of International Cosmic Ray Conference, p. 673, 1907.08018.

[27] Ž. Bošnjak, A. M. Brown, A. Carosi, et al., arXiv e-prints (2021), 2106.03621.

[28] B. Khiali, E. M. de Gouveia Dal Pino, and M. V. del Valle, Mon. Not. Roy. Astron. Soc. 449, 34 (2015), 1406.5664.

[29] J. Aleksić, L. A. Antonelli, P. Antoranz, et al. (MAGIC), The Astrophysical Journal 721, 843 (2010), 1005.0740 .

[30] M. L. Ahnen, S. Ansoldi, L. A. Antonelli, et al. (MAGIC), Mon. Not. Roy. Astron. Soc. 472, 3474 (2017), 1708.03689.

[31] J. Albert, E. Aliu, H. Anderhub, et al. (MAGIC), Astrophys. J. Lett. 665, L51 (2007), 0706.1505.

[32] A. Loh, S. Corbel, G. Dubus, et al., Mon. Not. Roy. Astron. Soc. 462, L111 (2016), 1607. 06239.

[33] M. L. Ahnen, S. Ansoldi, L. A. Antonelli, et al. (MAGIC), Mon. Not. Roy. Astron. Soc. 471, 1688 (2017), 1707.00887.

[34] J. Li, D. F. Torres, R. Y. Liu, M. Kerr, et al., Nature Astronomy 4, 1177 (2020), 2008. 10523.

[35] M. L. Ahnen et al. (MAGIC, H.E.S.S.), Astronomy and Astrophysics 612, A14 (2018), 1707.03658. 


\section{The Cherenkov Telescope Array Consortium July 2021 Authors}

H. Abdalla ${ }^{1}$, H. Abe ${ }^{2}$, S. Abe ${ }^{2}$, A. Abusleme ${ }^{3}$, F. Acero ${ }^{4}$, A. Acharyya ${ }^{5}$, V. Acín Portella ${ }^{6}$, K. Ackley ${ }^{7}$, R. Adam $^{8}$, C. Adams ${ }^{9}$, S.S. Adhikari ${ }^{10}$, I. Aguado-Ruesga ${ }^{11}$, I. Agudo ${ }^{12}$, R. Aguilera ${ }^{13}$, A. AguirreSantaella $^{14}$, F. Aharonian ${ }^{15}$, A. Alberdi ${ }^{12}$, R. Alfaro ${ }^{16}$, J. Alfaro ${ }^{3}$, C. Alispach ${ }^{17}$, R. Aloisio ${ }^{18}$, R. Alves Batista $^{19}$, J.-P. Amans ${ }^{20}$, L. Amati ${ }^{21}$, E. Amato ${ }^{22}$, L. Ambrogi ${ }^{18}$, G. Ambrosi ${ }^{23}$, M. Ambrosio ${ }^{24}$, R. Ammendola ${ }^{25}$, J. Anderson ${ }^{26}$, M. Anduze ${ }^{8}$, E.O. Angüner ${ }^{27}$, L.A. Antonelli ${ }^{28}$, V. Antonuccio ${ }^{29}$, P. Antoranz ${ }^{30}$, R. Anutarawiramkul ${ }^{31}$, J. Aragunde Gutierrez ${ }^{32}$, C. Aramo ${ }^{24}$, A. Araudo ${ }^{33,34}$, M. Araya ${ }^{35}$, A. Arbet-Engels ${ }^{36}$, C. Arcaro ${ }^{1}$, V. Arendt ${ }^{37}$, C. Armand ${ }^{38}$, T. Armstrong ${ }^{27}$, F. Arqueros ${ }^{11}$, L. Arrabito ${ }^{39}$, B. Arsioli ${ }^{40}$, M. Artero ${ }^{41}$, K. Asano ${ }^{2}$, Y. Ascasíbar ${ }^{14}$, J. Aschersleben ${ }^{42}$, M. Ashley ${ }^{43}$, P. Attinà ${ }^{44}$, P. Aubert ${ }^{45}$, C. B. Singh ${ }^{19}$, D. Baack ${ }^{46}$, A. Babic ${ }^{47}$, M. Backes ${ }^{48}$, V. Baena ${ }^{13}$, S. Bajtlik ${ }^{49}$, A. Baktash ${ }^{50}$, C. Balazs ${ }^{7}$, M. Balbo ${ }^{38}$, O. Ballester ${ }^{41}$, J. Ballet ${ }^{4}$, B. Balmaverde ${ }^{44}$, A. Bamba ${ }^{51}$, R. Bandiera ${ }^{22}$, A. Baquero Larriva ${ }^{11}$, P. Barai $^{19}$, C. Barbier ${ }^{45}$, V. Barbosa Martins ${ }^{52}$, M. Barcelo $^{53}$, M. Barkov ${ }^{54}$, M. Barnard ${ }^{1}$, L. Baroncelli ${ }^{21}$, U. Barres de Almeida ${ }^{40}$, J.A. Barrio ${ }^{11}$, D. Bastieri ${ }^{55}$, P.I. Batista ${ }^{52}$, I. Batkovic ${ }^{55}$, C. Bauer ${ }^{53}$, R. Bautista-González ${ }^{56}$, J. Baxter ${ }^{2}$, U. Becciani' ${ }^{29}$, J. Becerra González ${ }^{32}$, Y. Becherini ${ }^{57}$, G. Beck ${ }^{58}$, J. Becker Tjus ${ }^{59}$, W. Bednarek ${ }^{60}$, A. Belfiore ${ }^{61}$, L. Bellizzi ${ }^{62}$, R. Belmont ${ }^{4}$, W. Benbow ${ }^{63}$, D. Berge ${ }^{52}$, E. Bernardini ${ }^{52}$, M.I. Bernardos ${ }^{55}$, K. Bernlöhr ${ }^{53}$, A. Berti ${ }^{64}$, M. Berton ${ }^{65}$, B. Bertucci ${ }^{23}$, V. Beshley ${ }^{66}, N$. Bhatt ${ }^{67}$, S. Bhattacharyya ${ }^{67}$, W. Bhattacharyya ${ }^{52}$, S. Bhattacharyya ${ }^{68}$, B. Bi $^{69}$, G. Bicknell ${ }^{70}$, N. Biederbeck ${ }^{46}$, C. Bigongiari ${ }^{28}$, A. Biland ${ }^{36}$, R. Bird ${ }^{71}$, E. Bissaldi ${ }^{72}$, J. Biteau ${ }^{73}$, M. Bitossi ${ }^{74}$, O. Blanch ${ }^{41}$, M. Blank ${ }^{50}$, J. Blazek ${ }^{33}$, J. Bobin ${ }^{75}$, C. Boccato ${ }^{76}$, F. Bocchino ${ }^{77}$, C. Boehm ${ }^{78}$, M. Bohacova ${ }^{33}$, C. Boisson ${ }^{20}$, J. Boix ${ }^{41}$, J.-P. Bolle ${ }^{52}$, J. Bolmont ${ }^{79}$, G. Bonanno ${ }^{29}$, C. Bonavolontä ${ }^{24}$, L. Bonneau Arbeletche ${ }^{80}$, G. Bonnoli ${ }^{12}$, P. Bordas ${ }^{81}$, J. Borkowskii ${ }^{49}$, S. Bórquez ${ }^{35}$, R. Bose ${ }^{82}$, D. Bose ${ }^{83}$, Z. Bosnjak ${ }^{47}$, E. Bottacini ${ }^{55}$, M. Böttcher ${ }^{1}$, M.T. Botticella ${ }^{84}$, C. Boutonnet ${ }^{85}$, F. Bouyjou ${ }^{75}$, V. Bozhilov ${ }^{86}$, E. Bozzo ${ }^{38}$, L. Brahimi ${ }^{39}$, C. Braiding ${ }^{43}$, S. Brau-Nogué ${ }^{87}$, S. Breen ${ }^{78}$, J. Bregeon ${ }^{39}$, M. Breuhaus ${ }^{53}$, A. Brill ${ }^{9}$, W. Brisken ${ }^{88}$, E. Brocato ${ }^{28}$, A.M. Brown ${ }^{5}$, K. Brügge ${ }^{46}$, P. Brun ${ }^{89}$, P. Brun ${ }^{39}$, F. Brun ${ }^{89}$, L. Brunetti ${ }^{45}$, G. Brunetti ${ }^{90}$, P. Bruno ${ }^{29}$, A. Bruno ${ }^{91}$, A. Bruzzese ${ }^{6}$, N. Bucciantini2 ${ }^{22}$, J. Buckley ${ }^{82}$,

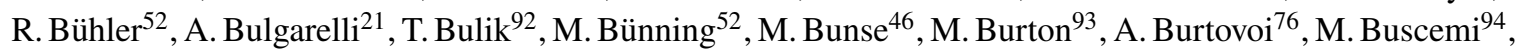
S. Buschjäger ${ }^{46}$, G. Busetto ${ }^{55}$, J. Buss ${ }^{46}$, K. Byrum ${ }^{26}$, A. Caccianiga ${ }^{95}$, F. Cadoux ${ }^{17}$, A. Calanducci ${ }^{29}$, C. Calderón ${ }^{3}$, J. Calvo Tovar ${ }^{32}$, R. Cameron ${ }^{96}$, P. Campaña ${ }^{35}$, R. Canestrari ${ }^{91}$, F. Cangemi ${ }^{79}$, B. Cantlay ${ }^{31}$, M. Capalbi ${ }^{91}$, M. Capasso ${ }^{9}$, M. Cappi ${ }^{21}$, A. Caproni ${ }^{97}$, R. Capuzzo-Dolcetta ${ }^{28}$, P. Caraveo ${ }^{61}$, V. Cárdenas ${ }^{98}$, L. Cardiel ${ }^{41}$, M. Cardillo ${ }^{99}$, C. Carlile ${ }^{100}$, S. Caroff ${ }^{45}$, R. Carosii ${ }^{74}$, A. Carosi ${ }^{17}$, E. Carquín ${ }^{35}$, M. Carrère ${ }^{39}$, J.-M. Casandjian ${ }^{4}$, S. Casanova ${ }^{101,53}$, E. Cascone ${ }^{84}$, F. Cassol ${ }^{27}$, A.J. Castro-Tirado ${ }^{12}$, F. Catalani ${ }^{102}$, O. Catalano ${ }^{91}$, D. Cauz ${ }^{103}$, A. Ceccanti ${ }^{64}$, C. Celestino Silva ${ }^{80}$, S. Celli ${ }^{18}$, K. Cerny ${ }^{104}$, M. Cerruti ${ }^{85}$, E. Chabanne ${ }^{45}$, P. Chadwick ${ }^{5}$, Y. Chai ${ }^{105}$, P. Chambery ${ }^{106}$, C. Champion ${ }^{85}$, S. Chandra ${ }^{1}$, S. Chaty ${ }^{4}$, A. $\mathrm{Chen}^{58}$, K. Cheng ${ }^{2}$, M. Chernyakova ${ }^{107}$, G. Chiaro ${ }^{61}$, A. Chiavassa ${ }^{64,108}$, M. Chikawa ${ }^{2}$, V.R. Chitnis ${ }^{109}$, J. Chudoba ${ }^{33}$, L. Chytka ${ }^{104}$, S. Cikota ${ }^{47}$, A. Circiello ${ }^{24,110}$, P. Clark ${ }^{5}$, M. Çolak ${ }^{41}$, E. Colombo ${ }^{32}$, J. Colome ${ }^{13}$, S. Colonges ${ }^{85}$, A. Comastri ${ }^{21}$, A. Compagnino ${ }^{91}$, V. Conforti ${ }^{21}$, E. Congiu ${ }^{95}$, R. Coniglione ${ }^{94}$, J. Conrad ${ }^{111}$, F. Conte ${ }^{53}$, J.L. Contreras ${ }^{11}$, P. Coppi ${ }^{112}$, R. Cornat ${ }^{8}$, J. Coronado-Blazquez ${ }^{14}$, J. Cortina ${ }^{113}$, A. Costa ${ }^{29}$, H. Costantini ${ }^{27}$, G. Cotter ${ }^{14}$, B. Courty ${ }^{85}$, S. Covino ${ }^{95}$, S. Crestan ${ }^{61}$, P. Cristofari ${ }^{20}$, R. Crocker ${ }^{70}$, J. Croston ${ }^{115}$, K. Cubuk ${ }^{93}$, O. Cuevas ${ }^{98}$, X. Cui ${ }^{2}$, G. Cusumano ${ }^{91}$, S. Cutini ${ }^{23}$, A. D’Aì ${ }^{91}$, G. D’Amico ${ }^{116}$, F. D’Ammando ${ }^{90}$, P. D’Avanzo ${ }^{95}$, P. Da Vela ${ }^{74}$, M. Dadina ${ }^{21}$, S. Dai ${ }^{17}$, M. Dalchenko ${ }^{17}$, M. Dall' Ora ${ }^{84}$, M.K. Daniel ${ }^{63}$, J. Dauguet ${ }^{85}$, I. Davids ${ }^{48}$, J. Davies ${ }^{114}$, B. Dawson ${ }^{118}$, A. De Angelis ${ }^{55}$, A.E. de Araújo Carvalho ${ }^{40}$, M. de Bony de Lavergne ${ }^{45}$, V. De Caprio ${ }^{84}$, G. De Cesare ${ }^{21}$, F. De Frondat ${ }^{20}$, E.M. de Gouveia Dal Pino ${ }^{19}$, I. de la Calle ${ }^{11}$, B. De Lotto ${ }^{103}$, A. De Luca ${ }^{61}$, D. De Martino ${ }^{84}$, R.M. de Menezes ${ }^{19}$, M. de Naurois ${ }^{8}$, E. de Oña Wilhelmi ${ }^{13}$, F. De Palma ${ }^{64}$, F. De Persio ${ }^{119}$, N. de Simone ${ }^{52}$, V. de Souza ${ }^{80}$, M. Del Santo ${ }^{91}$, M.V. del Valle ${ }^{19}$, E. Delagnes ${ }^{75}$, G. Deleglise ${ }^{45}$, M. Delfino Reznicek ${ }^{6}$, C. Delgado ${ }^{113}$, A.G. Delgado Giler $^{80}$, J. Delgado Mengual ${ }^{6}$, R. Della Ceca ${ }^{95}$, M. Della Valle ${ }^{84}$, D. della Volpe ${ }^{17}$, D. Depaoli ${ }^{64,108}$, D. Depouez ${ }^{27}$, J. Devin ${ }^{85}$, T. Di Girolamo ${ }^{24,110}$, C. Di Giulio ${ }^{25}$, A. Di Piano ${ }^{21}$, F. Di Pierro ${ }^{64}$, L. Di Venere $^{120}$, C. Díaz ${ }^{113}$, C. Díaz-Bahamondes ${ }^{3}$, C. Dib ${ }^{35}$, S. Diebold ${ }^{69}$, S. Digel ${ }^{96}$, R. Dima ${ }^{55}$, A. DjannatiAtai $^{85}$, J. Djuvsland ${ }^{116}$, A. Dmytriiev ${ }^{20}$, K. Docher ${ }^{9}$, A. Domínguez ${ }^{11}$, D. Dominis Prester ${ }^{121}$, A. Donath ${ }^{53}$, 
A. Donini ${ }^{41}$, D. Dorner ${ }^{122}$, M. Doro ${ }^{55}$, R.d.C. dos Anjos ${ }^{123}$, J.-L. Dournaux ${ }^{20}$, T. Downes ${ }^{107}$, G. Drake ${ }^{26}$, H. Drass ${ }^{3}$, D. Dravins ${ }^{100}$, C. Duangchan ${ }^{31}$, A. Duara ${ }^{124}$, G. Dubus ${ }^{125}$, L. Ducci ${ }^{69}$, C. Duffy ${ }^{124}$, D. Dumora ${ }^{106}$, K. Dundas Morå ${ }^{111}$, A. Durkalec ${ }^{126}$, V.V. Dwarkadas ${ }^{127}$, J. Ebr ${ }^{33}$, C. Eckner ${ }^{45}$, J. Eder ${ }^{105}$, A. Ederoclite ${ }^{19}$, E. Edy ${ }^{8}$, K. Egberts $^{128}$, S. Einecke ${ }^{118}$, J. Eisch ${ }^{129}$, C. Eleftheriadis ${ }^{130}$, D. Elsässer ${ }^{46}$, G. Emery ${ }^{17}$, D. Emmanoulopoulos ${ }^{115}$, J.-P. Ernenwein ${ }^{27}$, M. Errando ${ }^{82}$, P. Escarate ${ }^{35}$, J. Escudero ${ }^{12}$, C. Espinoza ${ }^{3}$, S. Ettori ${ }^{21}$, A. Eungwanichayapant ${ }^{31}$, P. Evans ${ }^{124}$, C. Evoli ${ }^{18}$, M. Fairbairn ${ }^{131}$, D. Falceta-Goncalves ${ }^{132}$, A. Falcone ${ }^{133}$, V. Fallah Ramazani ${ }^{65}$, R. Falomo ${ }^{76}$, K. Farakos ${ }^{134}$, G. Fasola ${ }^{20}$, A. Fattorini ${ }^{46}$, Y. Favre ${ }^{17}$, R. Fedora ${ }^{135}$, E. Fedorova ${ }^{136}$, S. Fegan $^{8}$, K. Feijen ${ }^{118}$, Q. Feng ${ }^{9}$, G. Ferrand ${ }^{54}$, G. Ferrara ${ }^{94}$, O. Ferreira ${ }^{8}$, M. Fesquet ${ }^{75}$, E. Fiandrini ${ }^{23}$, A. Fiasson ${ }^{45}$, M. Filipovic ${ }^{117}$, D. Fink ${ }^{105}$, J.P. Finley ${ }^{137}$, V. Fioretti ${ }^{21}$, D.F.G. Fiorillo ${ }^{24,110}$, M. Fiorini ${ }^{61}$, S. Flis ${ }^{52}$, H. Flores ${ }^{20}$, L. Foffano ${ }^{17}$, C. Föhr ${ }^{53}$, M.V. Fonseca ${ }^{11}$, L. Font ${ }^{138}$, G. Fontaine ${ }^{8}$, O. Fornieri ${ }^{52}$, P. Fortin ${ }^{63}$, L. Fortson ${ }^{88}$, N. Fouque ${ }^{45}$, A. Fournier ${ }^{106}$, B. Fraga ${ }^{40}$, A. Franceschini ${ }^{76}$, F.J. Franco ${ }^{30}$, A. Franco Ordovas ${ }^{32}$, L. Freixas Coromina ${ }^{113}$, L. Fresnillo ${ }^{30}$, C. Fruck ${ }^{105}$, D. Fugazza ${ }^{95}$, Y. Fujikawa ${ }^{139}$, Y. Fujita ${ }^{2}$, S. Fukami ${ }^{2}$, Y. Fukazawa ${ }^{140}$, Y. Fukui ${ }^{141}$, D. Fulla ${ }^{52}$, S. Funk ${ }^{142}$, A. Furniss ${ }^{143}$, O. Gabella ${ }^{39}$, S. Gabici ${ }^{85}$, D. Gaggero ${ }^{14}$, G. Galanti ${ }^{61}$, G. Galaz ${ }^{3}$, P. Galdemard ${ }^{144}$, Y. Gallant ${ }^{39}$, D. Galloway ${ }^{7}$, S. Gallozzi ${ }^{28}$, V. Gammaldi ${ }^{14}$, R. Garcia ${ }^{41}$, E. Garcia ${ }^{45}$, E. García ${ }^{13}$, R. Garcia López ${ }^{32}$, M. Garczarczyk ${ }^{52}$, F. Gargano ${ }^{120}$, C. Gargano ${ }^{91}$, S. Garozzo ${ }^{29}$, D. Gascon ${ }^{81}$, T. Gasparetto ${ }^{145}$, D. Gasparrini2 ${ }^{25}$, H. Gasparyan ${ }^{52}$, M. Gaug ${ }^{138}$, N. Geffroy ${ }^{45}$, A. Gent ${ }^{146}$, S. Germani ${ }^{76}$, L. Gesa ${ }^{13}$, A. Ghalumyan ${ }^{147}$, A. Ghedina ${ }^{148}$, G. Ghirlanda ${ }^{95}$, F. Gianotti ${ }^{21}$, S. Giarrusso ${ }^{91}$, M. Giarrusso ${ }^{94}$, G. Giavitto ${ }^{52}$, B. Giebels ${ }^{8}$, N. Giglietto ${ }^{72}$, V. Gika ${ }^{134}$, F. Gillardo ${ }^{45}$, R. Gimenes ${ }^{19}$, F. Giordano ${ }^{149}$, G. Giovannini ${ }^{90}$, E. Giro ${ }^{76}$, M. Giroletti ${ }^{90}$, A. Giuliani ${ }^{61}$, L. Giunti ${ }^{85}$, M. Gjaja ${ }^{9}$, J.-F. Glicenstein ${ }^{89}$, P. Gliwny ${ }^{60}$, N. Godinovic ${ }^{150}$, H. Göksu ${ }^{53}$, P. Goldoni ${ }^{85}$, J.L. Gómez ${ }^{12}$, G. Gómez-Vargas ${ }^{3}$, M.M. González ${ }^{16}$, J.M. González ${ }^{151}$, K.S. Gothe ${ }^{109}$, D. Götz ${ }^{4}$, J. Goulart Coelho ${ }^{123}$, K. Gourgouliatos ${ }^{5}$, T. Grabarczyk ${ }^{152}$, R. Graciani ${ }^{81}$, P. Grandi ${ }^{21}$, G. Grasseau ${ }^{8}$, D. Grasso ${ }^{74}$, A.J. Green ${ }^{78}$, D. Green ${ }^{105}$, J. Green ${ }^{28}$, T. Greenshaw ${ }^{153}$, I. Grenier ${ }^{4}$, P. Grespan ${ }^{55}$, A. Grillo ${ }^{29}$, M.-H. Grondin ${ }^{106}$, J. Grube ${ }^{131}$, V. Guarino ${ }^{26}$, B. Guest ${ }^{37}$, O. Gueta ${ }^{52}$, M. Gündüz ${ }^{59}$, S. Gunji ${ }^{154}$, A. Gusdorf ${ }^{20}$, G. Gyuk ${ }^{155}$, J. Hackfeld ${ }^{59}$, D. Hadasch ${ }^{2}$, J. Haga ${ }^{139}$, L. Hagge ${ }^{52}$, A. Hahn ${ }^{105}$, J.E. Hajlaoui ${ }^{85}$, H. Hakobyan ${ }^{35}$, A. Halim ${ }^{89}$, P. Hamal ${ }^{33}$, W. Hanlon ${ }^{63}$, S. Hara ${ }^{156}$, Y. Harada ${ }^{157}$, M.J. Hardcastle ${ }^{158}$, M. Harvey ${ }^{5}$, K. Hashiyama ${ }^{2}$, T. Hassan Collado ${ }^{113}$, T. Haubold ${ }^{105}$, A. Haupt ${ }^{52}$, U.A. Hautmann ${ }^{159}$, M. Havelka ${ }^{33}$, K. Hayashi ${ }^{141}$, K. Hayashi ${ }^{160}$, M. Hayashida ${ }^{161}$, H. He ${ }^{54}$, L. Heckmann ${ }^{105}$, M. Heller ${ }^{17}$, J.C. Helo ${ }^{35}$, F. Henault ${ }^{125}$, G. Henri ${ }^{125}$, G. Hermann ${ }^{53}$, R. Hermel ${ }^{45}$, S. Hernández Cadena ${ }^{16}$, J. Herrera Llorente ${ }^{32}$, A. Herrero ${ }^{32}$, O. Hervet ${ }^{143}$, J. Hinton ${ }^{53}$, A. Hiramatsu ${ }^{157}$, N. Hiroshima ${ }^{54}$, K. Hirotani ${ }^{2}$, B. Hnatyk ${ }^{136}$, R. Hnatyk ${ }^{136}$, J.K. Hoang ${ }^{11}$, D. Hoffmann ${ }^{27}$, W. Hofmann ${ }^{53}$, C. Hoischen ${ }^{128}$, J. Holder ${ }^{162}$, M. Holler ${ }^{163}$, B. Hona ${ }^{164}$, D. Horan ${ }^{8}$, J. Hörandel ${ }^{165}$, D. Horns ${ }^{50}$, P. Horvath ${ }^{104}$, J. Houles ${ }^{27}$, T. Hovatta ${ }^{65}$, M. Hrabovsky ${ }^{104}$, D. Hrupec ${ }^{166}$, Y. Huang ${ }^{135}$, J.-M. Huet ${ }^{20}$, G. Hughes ${ }^{159}$, D. Hui ${ }^{2}$, G. Hull ${ }^{73}$, T.B. Humensky ${ }^{9}$, M. Hütten ${ }^{105}$, R. Iaria ${ }^{77}$, M. Iarlori ${ }^{18}$, J.M. Illa $^{41}$, R. Imazawa ${ }^{140}$, D. Impiombato ${ }^{91}$, T. Inada ${ }^{2}$, F. Incardona ${ }^{29}$, A. Ingallinera ${ }^{29}$, Y. Inome ${ }^{2}$, S. Inoue ${ }^{54}$, T. Inoue ${ }^{141}$, Y. Inoue ${ }^{167}$, A. Insolia ${ }^{120,94}$, F. Iocco ${ }^{24,110}$, K. Ioka ${ }^{168}$, M. Ionica $^{23}$, M. Iori ${ }^{119}$, S. Iovenitti ${ }^{95}$, A. Iriarte ${ }^{16}$, K. Ishio ${ }^{105}$, W. Ishizaki ${ }^{168}$, Y. Iwamura ${ }^{2}$, C. Jablonski ${ }^{105}$, J. Jacquemier ${ }^{45}$ M. Jacquemont ${ }^{45}$, M. Jamrozy ${ }^{169}$, P. Janecek ${ }^{33}$, F. Jankowsky ${ }^{170}$, A. Jardin-Blicq ${ }^{31}$, C. Jarnot ${ }^{87}$, P. Jean ${ }^{87}$, I. Jiménez Martínez ${ }^{113}$, W. Jin ${ }^{171}$, L. Jocou ${ }^{125}$, N. Jordana ${ }^{172}$, M. Josselin ${ }^{73}$, L. Jouvin ${ }^{41}$, I. Jung-Richardt ${ }^{142}$, F.J.P.A. Junqueira ${ }^{19}$, C. Juramy-Gilles ${ }^{79}$, J. Jurysek ${ }^{38}$, P. Kaaret ${ }^{173}$, L.H.S. Kadowaki ${ }^{19}$, M. Kagaya ${ }^{2}$, O. Kalekin ${ }^{142}$, R. Kankanyan ${ }^{53}$, D. Kantzas ${ }^{174}$, V. Karas ${ }^{34}$, A. Karastergiou ${ }^{114}$, S. Karkar ${ }^{79}$, E. Kasai ${ }^{48}$, J. Kasperek ${ }^{175}$, H. Katagiri ${ }^{176}$, J. Kataoka ${ }^{177}$, K. Katarzyński ${ }^{178}$, S. Katsuda ${ }^{179}$, U. Katz ${ }^{142}$, N. Kawanaka ${ }^{180}$, D. Kazanas ${ }^{130}$, D. Kerszberg ${ }^{41}$, B. Khélifi ${ }^{85}$, M.C. Kherlakian ${ }^{52}$, T.P. Kian ${ }^{181}$, D.B. Kieda ${ }^{164}$, T. Kihm ${ }^{53}$, S. Kim ${ }^{3}$, S. Kimeswenger ${ }^{163}$, S. Kisaka ${ }^{140}$, R. Kissmann ${ }^{163}$, R. Kleijwegt ${ }^{135}$, T. Kleiner ${ }^{52}$, G. Kluge ${ }^{10}$, W. Kluźniak ${ }^{49}$, J. Knapp ${ }^{52}$, J. Knödlseder ${ }^{87}$, A. Kobakhidze ${ }^{78}$, Y. Kobayashi ${ }^{2}$, B. Koch ${ }^{3}$, J. Kocot ${ }^{152}$, K. Kohri ${ }^{182}$, K. Kokkotas ${ }^{69}$, N. Komin ${ }^{58}$, A. Kong ${ }^{2}$, K. Kosack ${ }^{4}$, G. Kowal ${ }^{132}$, F. Krack ${ }^{52}$, M. Krause ${ }^{52}$, F. Krennrich ${ }^{129}$, M. Krumholz ${ }^{70}$, H. Kubo ${ }^{180}$, V. Kudryavtsev ${ }^{183}$, S. Kunwar ${ }^{53}$, Y. Kuroda ${ }^{139}$, J. Kushida ${ }^{157}$, P. Kushwaha ${ }^{19}$, A. La Barbera ${ }^{91}$, N. La Palombara ${ }^{61}$, V. La Parola ${ }^{91}$, G. La Rosa ${ }^{91}$, R. Lahmann ${ }^{142}$, G. Lamanna ${ }^{45}$, A. Lamastra ${ }^{28}$, M. Landoni ${ }^{95}$, D. Landriu ${ }^{4}$, R.G. Lang ${ }^{80}$, J. Lapington ${ }^{124}$, P. Laporte ${ }^{20}$, 
P. Lason ${ }^{152}$, J. Lasuik ${ }^{37}$, J. Lazendic-Galloway ${ }^{7}$, T. Le Flour ${ }^{45}$, P. Le Sidaner ${ }^{20}$, S. Leach ${ }^{124}$, A. Leckngam ${ }^{31}$, S.-H. Lee ${ }^{180}$, W.H. Lee ${ }^{16}$, S. Lee ${ }^{118}$, M.A. Leigui de Oliveira ${ }^{184}$, A. Lemière ${ }^{85}$, M. Lemoine-Goumard ${ }^{106}$, J.-P. Lenain ${ }^{79}$, F. Leone ${ }^{94,185}$, V. Leray ${ }^{8}$, G. Leto ${ }^{29}$, F. Leuschner ${ }^{69}$, C. Levy ${ }^{79,20}$, R. Lindemann ${ }^{52}$, E. Lindfors ${ }^{65}$, L. Linhoff ${ }^{46}$, I. Liodakis ${ }^{65}$, A. Lipniacka ${ }^{116}$, S. Lloyd ${ }^{5}$, M. Lobo ${ }^{113}$, T. Lohse ${ }^{186}$, S. Lombardi ${ }^{28}$, F. Longo ${ }^{145}$, A. López-Oramas ${ }^{32}$, M. López ${ }^{11}$, R. López-Coto ${ }^{55}$, S. Loporchio ${ }^{149}$, F. Louis ${ }^{75}$, M. Louys ${ }^{20}$, F. Lucarelli ${ }^{28}$, D. Lucchesi ${ }^{55}$, H. Ludwig Boudi ${ }^{39}$, P.L. Luque-Escamilla ${ }^{56}$, E. Lyard ${ }^{38}$, M.C. Maccarone ${ }^{91}$, T. Maccarone $^{187}$, E. Mach $^{101}$, A.J. Maciejewski ${ }^{188}$, J. Mackey ${ }^{15}$, G.M. Madejski ${ }^{96}$, P. Maeght ${ }^{39}$, C. Maggio ${ }^{138}$, G. Maier ${ }^{52}$, A. Majczyna ${ }^{126}$, P. Majumdar ${ }^{83,2}$, M. Makariev ${ }^{189}$, M. Mallamaci ${ }^{55}$, R. Malta Nunes de Almeida ${ }^{184}$, S. Maltezos ${ }^{134}$, D. Malyshev ${ }^{142}$, D. Malyshev ${ }^{69}$, D. Mandat ${ }^{33}$, G. Maneva ${ }^{189}$, M. Manganaro ${ }^{121}$, G. Manicò ${ }^{94}$, P. Manigot ${ }^{8}$, K. Mannheim ${ }^{122}$, N. Maragos ${ }^{134}$, D. Marano ${ }^{29}$, M. Marconi ${ }^{84}$, A. Marcowith ${ }^{39}$, M. Marculewicz ${ }^{190}$, B. Marčun ${ }^{68}$, J. Marín ${ }^{98}$, N. Marinello ${ }^{55}$, P. Marinos ${ }^{118}$, M. Mariotti ${ }^{55}$, S. Markoff ${ }^{174}$, P. Marquez ${ }^{41}$, G. Marsella ${ }^{94}$, J. Martí56, J.-M. Martin ${ }^{20}$, P. Martin $^{87}$, O. Martinez ${ }^{30}$, M. Martínez ${ }^{41}$, G. Martínez ${ }^{113}$, O. Martínez ${ }^{41}$, H. Martínez-Huerta ${ }^{80}$, C. Marty ${ }^{87}$, R. Marx $^{53}$, N. Masetti ${ }^{21,151}$, P. Massimino ${ }^{29}$, A. Mastichiadis ${ }^{191}$, H. Matsumoto ${ }^{167}$, N. Matthews ${ }^{164}$, G. Maurin ${ }^{45}$, W. Max-Moerbeck ${ }^{192}$, N. Maxted ${ }^{43}$, D. Mazin²,105, M.N. Mazziotta ${ }^{120}$, S.M. Mazzola ${ }^{77}$, J.D. Mbarubucyeye ${ }^{52}$, L. Mc Comb ${ }^{5}$, I. McHardy ${ }^{115}$, S. McKeague ${ }^{107}$, S. McMuldroch ${ }^{63}$, E. Medina ${ }^{64}$, D. Medina Miranda ${ }^{17}$, A. Melandri ${ }^{95}$, C. Melioli ${ }^{19}$, D. Melkumyan ${ }^{52}$, S. Menchiari ${ }^{62}$, S. Mender ${ }^{46}$, S. Mereghetti ${ }^{61}$, G. Merino Arévalo ${ }^{6}$, E. Mestre ${ }^{13}$, J.-L. Meunier ${ }^{79}$, T. Meures ${ }^{135}$, M. Meyer ${ }^{142}$, S. Micanovic ${ }^{121}$, M. Miceli ${ }^{77}$, M. Michailidis ${ }^{69}$, J. Michałowski ${ }^{101}$, T. Miener ${ }^{11}$, I. Mievre ${ }^{45}$, J. Miller ${ }^{35}$, I.A. Minaya ${ }^{153}$, T. Mineo ${ }^{91}$, M. Minev ${ }^{189}$, J.M. Miranda ${ }^{30}$, R. Mirzoyan ${ }^{105}$, A. Mitchell ${ }^{36}$, T. Mizuno ${ }^{193}$, B. Mode ${ }^{135}$, R. Moderski ${ }^{49}$, L. Mohrmann ${ }^{142}$, E. Molina ${ }^{81}$, E. Molinari ${ }^{148}$, T. Montaruli ${ }^{17}$, I. Monteiro $^{45}$, C. Moore ${ }^{124}$, A. Moralejo ${ }^{41}$, D. Morcuende-Parrilla ${ }^{11}$, E. Moretti ${ }^{41}$, L. Morganti ${ }^{64}$, K. Mori ${ }^{194}$, P. Moriarty ${ }^{15}$, K. Morik ${ }^{46}$, G. Morlino ${ }^{22}$, P. Morris ${ }^{114}$, A. Morselli25, K. Mosshammer ${ }^{52}$, P. Moya ${ }^{192}$, R. Mukherjee ${ }^{9}$, J. Muller ${ }^{8}$, C. Mundell ${ }^{172}$, J. Mundet ${ }^{41}$, T. Murach ${ }^{52}$, A. Muraczewski ${ }^{49}$, H. Muraishi ${ }^{195}$,

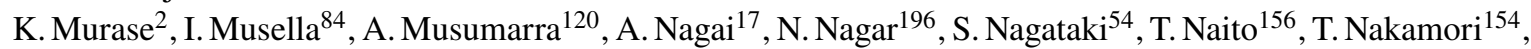
K. Nakashima ${ }^{142}$, K. Nakayama ${ }^{51}$, N. Nakhjiri ${ }^{13}$, G. Naletto ${ }^{55}$, D. Naumann ${ }^{52}$, L. Nava ${ }^{95}$, R. Navarro ${ }^{174}$, M.A. Nawaz ${ }^{132}$, H. Ndiyavala ${ }^{1}$, D. Neise ${ }^{36}$, L. Nellen ${ }^{16}$, R. Nemmen ${ }^{19}$, M. Newbold ${ }^{164}$, N. Neyroud ${ }^{45}$, K. Ngernphat ${ }^{31}$, T. Nguyen Trung ${ }^{73}$, L. Nicastro ${ }^{21}$, L. Nickel ${ }^{46}$, J. Niemiec ${ }^{101}$, D. Nieto ${ }^{11}$, M. Nievas ${ }^{32}$, C. Nigro ${ }^{41}$, M. Nikołajuk ${ }^{190}$, D. Ninci ${ }^{41}$, K. Nishijima ${ }^{157}$, K. Noda ${ }^{2}$, Y. Nogami ${ }^{176}$, S. Nolan ${ }^{5}$, R. Nomura ${ }^{2}$, R. Norris ${ }^{117}$, D. Nosek ${ }^{197}$, M. Nöthe ${ }^{46}$, B. Novosyadlyj ${ }^{198}$, V. Novotny ${ }^{197}$, S. Nozaki ${ }^{180}$, F. Nunio ${ }^{144}$, P. O’Brien ${ }^{124}$, K. Obara ${ }^{176}$, R. Oger ${ }^{85}$, Y. Ohira ${ }^{51}$, M. Ohishi ${ }^{2}$, S. Ohm ${ }^{52}$, Y. Ohtani ${ }^{2}$, T. Oka ${ }^{180}$, N. Okazaki ${ }^{2}$, A. Okumura ${ }^{139,199}$, J.-F. Olive ${ }^{87}$, C. Oliver $^{30}$, G. Olivera ${ }^{52}$, B. Olmi ${ }^{22}$, R.A. Ong ${ }^{71}$, M. Orienti ${ }^{90}$, R. Orito ${ }^{200}$, M. Orlandini ${ }^{21}$, S. Orlando ${ }^{77}$, E. Orlando ${ }^{145}$, J.P. Osborne ${ }^{124}$, M. Ostrowski ${ }^{169}$, N. Otte ${ }^{146}$, E. Ovcharov ${ }^{86}$, E. Owen ${ }^{2}$, I. Oya ${ }^{159}$, A. Ozieblo ${ }^{152}$, M. Padovani ${ }^{22}$, I. Pagano ${ }^{29}$, A. Pagliaro ${ }^{91}$, A. Paizis ${ }^{61}$, M. Palatiello ${ }^{145}$, M. Palatka ${ }^{33}$, E. Palazzi ${ }^{21}$, J.-L. Panazol ${ }^{45}$, D. Paneque ${ }^{105}$, B. Panes ${ }^{3}$, S. Panny ${ }^{163}$, F.R. Pantaleo ${ }^{72}$, M. Panter ${ }^{53}$, R. Paoletti ${ }^{62}$, M. Paolillo ${ }^{24,110}$, A. Papitto ${ }^{28}$, A. Paravac ${ }^{122}$, J.M. Paredes ${ }^{81}$, G. Pareschi ${ }^{95}$, N. Park ${ }^{127}$, N. Parmiggiani ${ }^{21}$, R.D. Parsons ${ }^{186}$, P. Paśko ${ }^{201}$, S. Patel ${ }^{52}$, B. Patricelli28, G. Pauletta ${ }^{103}$, L. Pavletić ${ }^{121}$, S. Pavy $^{8}$, A. Pe'er ${ }^{105}$, M. Pech ${ }^{33}$, M. Pecimotika ${ }^{121}$, M.G. Pellegriti ${ }^{120}$, P. Peñil Del $\mathrm{Campo}^{11}$, M. Penno ${ }^{52}$, A. Pepato ${ }^{55}$, S. Perard ${ }^{106}$, C. Perennes ${ }^{55}$, G. Peres ${ }^{77}$, M. Peresano ${ }^{4}$, A. PérezAguilera $^{11}$, J. Pérez-Romero ${ }^{14}$, M.A. Pérez-Torres ${ }^{12}$, M. Perri ${ }^{28}$, M. Persic ${ }^{103}$, S. Petrera ${ }^{18}$, P.-O. Petrucci ${ }^{125}$, O. Petruk ${ }^{66}$, B. Peyaud ${ }^{89}$, K. Pfrang ${ }^{52}$, E. Pian ${ }^{21}$, G. Piano ${ }^{99}$, P. Piatteli ${ }^{94}$, E. Pietropaolo ${ }^{18}$, R. Pillera ${ }^{149}$, B. Pilszyk ${ }^{101}$, D. Pimentel ${ }^{202}$, F. Pintore ${ }^{91}$, C. Pio García ${ }^{41}$, G. Pirola ${ }^{64}$, F. Piron ${ }^{39}$, A. Pisarski ${ }^{190}$, S. Pita ${ }^{85}$, M. Pohl ${ }^{128}$, V. Poireau ${ }^{45}$, P. Poledrelli ${ }^{159}$, A. Pollo ${ }^{126}$, M. Polo ${ }^{113}$, C. Pongkitivanichkul ${ }^{31}$,

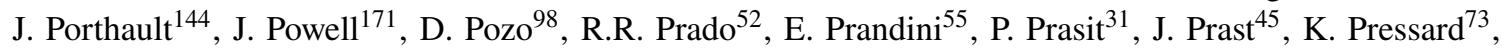
G. Principe ${ }^{90}$, C. Priyadarshi ${ }^{41}$, N. Produit ${ }^{38}$, D. Prokhorov ${ }^{174}$, H. Prokoph ${ }^{52}$, M. Prouza ${ }^{33}$, H. Przybilski $^{101}$, E. Pueschel ${ }^{52}$, G. Pühlhofer ${ }^{69}$, I. Puljak ${ }^{150}$, M.L. Pumo ${ }^{94}$, M. Punch ${ }^{85,57}$, F. Queiroz ${ }^{203}$, J. Quinn ${ }^{204}$, A. Quirrenbach ${ }^{170}$, S. Rainò ${ }^{149}$, P.J. Rajda ${ }^{175}$, R. Rando ${ }^{55}$, S. Razzaque ${ }^{205}$, E. Rebert ${ }^{20}$, S. Recchia ${ }^{85}$, P. Reichherzer ${ }^{59}$, O. Reimer ${ }^{163}$, A. Reimer ${ }^{163}$, A. Reisenegger ${ }^{3,206}$, Q. Remy ${ }^{53}$, M. Renaud ${ }^{39}$, T. Reposeur ${ }^{106}$, B. Reville ${ }^{53}$, J.-M. Reymond ${ }^{75}$, J. Reynolds ${ }^{15}$, W. Rhode ${ }^{46}$, D. Ribeiro ${ }^{9}$, M. Ribó ${ }^{81}$, G. Richards ${ }^{162}$, 
T. Richtler ${ }^{196}$, J. Rico ${ }^{41}$, F. Rieger ${ }^{53}$, L. Riitano ${ }^{135}$, V. Ripepi ${ }^{84}$, M. Riquelme ${ }^{192}$, D. Riquelme ${ }^{35}$, S. Rivoire ${ }^{39}$, V. Rizi ${ }^{18}$, E. Roache ${ }^{63}$, B. Röben ${ }^{159}$, M. Roche ${ }^{106}$, J. Rodriguez ${ }^{4}$, G. Rodriguez Fernandez ${ }^{25}$, J.C. Rodriguez Ramirez ${ }^{19}$, J.J. Rodríguez Vázquez ${ }^{113}$, F. Roepke ${ }^{170}$, G. Rojas ${ }^{207}$, L. Romanato ${ }^{55}$, P. Romano ${ }^{95}$, G. Romeo ${ }^{29}$, F. Romero Lobato ${ }^{11}$, C. Romoli ${ }^{53}$, M. Roncadelli ${ }^{103}$, S. Ronda ${ }^{30}$, J. Rosado ${ }^{11}$, A. Rosales de Leon ${ }^{5}$, G. Rowell ${ }^{118}$, B. Rudak ${ }^{49}$, A. Rugliancich ${ }^{74}$, J.E. Ruíz del Mazo ${ }^{12}$, W. Rujopakarn ${ }^{31}$, C. Rulten ${ }^{5}$, C. Russell ${ }^{3}$, F. Russo ${ }^{21}$, I. Sadeh ${ }^{52}$, E. Sæther Hatlen ${ }^{10}$, S. Safi-Harb ${ }^{37}$, L. Saha ${ }^{11}$, P. Saha ${ }^{208}$, V. Sahakian ${ }^{147}$, S. Sailer ${ }^{53}$, T. Saito ${ }^{2}$, N. Sakaki ${ }^{54}$, S. Sakurai ${ }^{2}$, F. Salesa Greus ${ }^{101}$, G. Salina ${ }^{25}$, H. Salzmann ${ }^{69}$, D. Sanchez ${ }^{45}$, M. Sánchez-Conde ${ }^{14}$, H. Sandaker ${ }^{10}$, A. Sandoval ${ }^{16}$, P. Sangiorgi ${ }^{91}$, M. Sanguillon ${ }^{39}$, H. Sano $^{2}$, M. Santander ${ }^{171}$, A. Santangelo ${ }^{69}$, E.M. Santos ${ }^{202}$, R. Santos-Lima ${ }^{19}$, A. Sanuy ${ }^{81}$, L. Sapozhnikov ${ }^{96}$, T. Saric ${ }^{150}$, S. Sarkar ${ }^{14}$, H. Sasaki ${ }^{157}$, N. Sasaki ${ }^{179}$, K. Satalecka ${ }^{52}$, Y. Sato ${ }^{209}$, F.G. Saturni ${ }^{28}$, M. Sawada ${ }^{54}$, U. Sawangwit ${ }^{31}$, J. Schaefer ${ }^{142}$, A. Scherer ${ }^{3}$, J. Scherpenberg ${ }^{105}$, P. Schipani ${ }^{84}$, B. Schleicher ${ }^{122}$, J. Schmoll ${ }^{5}$, M. Schneider ${ }^{143}$, H. Schoorlemmer ${ }^{53}$, P. Schovanek ${ }^{33}$, F. Schussler ${ }^{89}$, B. Schwab ${ }^{142}$, U. Schwanke ${ }^{186}$, J. Schwarz ${ }^{95}$, T. Schweizer ${ }^{105}$, E. Sciacca ${ }^{29}$, S. Scuderi ${ }^{61}$, M. Seglar Arroyo ${ }^{45}$, A. Segreto ${ }^{91}$, I. Seitenzahl ${ }^{43}$, D. Semikoz ${ }^{85}$, O. Sergijenko ${ }^{136}$, J.E. Serna Franco ${ }^{16}$, M. Servillat ${ }^{20}$, K. Seweryn $^{201}$, V. Sguera ${ }^{21}$, A. Shalchi ${ }^{37}$, R.Y. Shang ${ }^{71}$, P. Sharma ${ }^{73}$, R.C. Shellard ${ }^{40}$, L. Sidoli ${ }^{61}$, J. Sieiro ${ }^{81}$, H. Siejkowski ${ }^{152}$, J. Silk ${ }^{114}$, A. Sillanpää ${ }^{65}$, B.B. Singh ${ }^{109}$, K.K. Singh ${ }^{210}$, A. Sinha ${ }^{39}$, C. Siqueira ${ }^{80}$, G. Sironi ${ }^{95}$, J. Sitarek ${ }^{60}$, P. Sizun ${ }^{75}$, V. Sliusar ${ }^{38}$, A. Slowikowska ${ }^{178}$, D. Sobczyńska ${ }^{60}$, R.W. Sobrinho ${ }^{184}$, H. Sol $^{20}$, G. Sottile ${ }^{91}$, H. Spackman ${ }^{114}$, A. Specovius ${ }^{142}$, S. Spencer ${ }^{114}$, G. Spengler ${ }^{186}$, D. Spiga ${ }^{95}$, A. Spolon ${ }^{55}$, W. Springer ${ }^{164}$, A. Stamerra ${ }^{28}$, S. Stanic ${ }^{68}$, R. Starling ${ }^{124}$, Ł. Stawarz ${ }^{169}$, R. Steenkamp ${ }^{48}$, S. Stefanik ${ }^{197}$, C. Stegmann ${ }^{128}$, A. Steiner ${ }^{52}$, S. Steinmass ${ }^{53}$, C. Stella ${ }^{103}$, C. Steppa ${ }^{128}$, R. Sternberger ${ }^{52}$, M. Sterzel ${ }^{152}$, C. Stevens ${ }^{135}$, B. Stevenson ${ }^{71}$, T. Stolarczyk ${ }^{4}$, G. Stratta ${ }^{21}$, U. Straumann ${ }^{208}$, J. Strišković ${ }^{166}$, M. Strzys ${ }^{2}$, R. Stuik ${ }^{174}$, M. Suchenek ${ }^{211}$, Y. Suda ${ }^{140}$, Y. Sunada ${ }^{179}$, T. Suomijarvi ${ }^{73}$, T. Suric ${ }^{212}$, P. Sutcliffe ${ }^{153}$, H. Suzuki $^{213}$, P. Świerk ${ }^{101}$, T. Szepieniec ${ }^{152}$, A. Tacchini ${ }^{21}$, K. Tachihara ${ }^{141}$, G. Tagliaferri ${ }^{95}, \mathrm{H}$. Tajima $^{139}$, N. Tajima ${ }^{2}$, D. Tak ${ }^{52}$, K. Takahashi $^{214}$, H. Takahashi ${ }^{140}$, M. Takahashi ${ }^{2}$, M. Takahashi ${ }^{2}$ J. Takata $^{2}$, R. Takeishi ${ }^{2}$, T. Tam ${ }^{2}$, M. Tanaka ${ }^{182}$, T. Tanaka $^{213}$, S. Tanaka ${ }^{209}$, D. Tateishi ${ }^{179}$, M. Tavani ${ }^{99}$,F. Tavecchio ${ }^{95}$, T. Tavernier ${ }^{89}$, L. Taylor ${ }^{135}$, A. Taylor ${ }^{52}$, L.A. Tejedor ${ }^{11}$, P. Temnikov ${ }^{189}$, Y. Terada ${ }^{179}$, K. Terauchi ${ }^{180}$, J.C. Terrazas ${ }^{192}$, R. Terrier ${ }^{85}$, T. Terzic ${ }^{121}$, M. Teshima ${ }^{105,2}$,

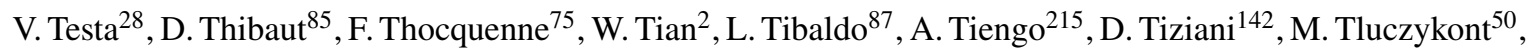
C.J. Todero Peixoto ${ }^{102}$, F. Tokanai ${ }^{154}$, K. Toma ${ }^{160}$, L. Tomankova ${ }^{142}$, J. Tomastik ${ }^{104}$, D. Tonev ${ }^{189}$, M. Tornikoski ${ }^{216}$,

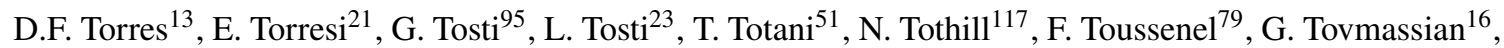

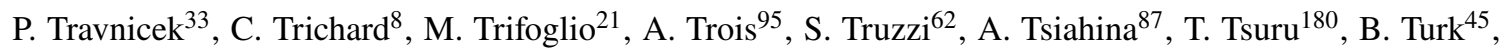
A. Tutone ${ }^{91}$, Y. Uchiyama ${ }^{161}$, G. Umana ${ }^{29}$, P. Utayarat ${ }^{31}$, L. Vaclavek ${ }^{104}$, M. Vacula ${ }^{104}$, V. Vagellii ${ }^{23,217}$, F. Vagnetti ${ }^{25}$, F. Vakilii ${ }^{218}$, J.A. Valdivia ${ }^{192}$, M. Valentino ${ }^{24}$, A. Valio ${ }^{19}$, B. Vallage ${ }^{89}$, P. Vallania ${ }^{44,64}$, J.V. Valverde Quispe ${ }^{8}$, A.M. Van den Berg ${ }^{42}$, W. van Driel ${ }^{20}$, C. van Eldik $^{142}$, C. van Rensburg ${ }^{1}$, B. van Soelen $^{210}$, J. Vandenbroucke ${ }^{135}$, J. Vanderwalt ${ }^{1}$, G. Vasileiadis ${ }^{39}$, V. Vassiliev ${ }^{71}$, M. Vázquez Acosta ${ }^{32}$, M. Vecchi ${ }^{42}$, A. Vega ${ }^{98}$, J. Veh ${ }^{142}$, P. Veitch ${ }^{118}$, P. Venault ${ }^{75}$, C. Venter ${ }^{1}$, S. Ventura ${ }^{62}$, S. Vercellone ${ }^{95}$, S. Vergani ${ }^{20}$, V. Verguilov ${ }^{189}$, G. Verna ${ }^{27}$, S. Vernetto ${ }^{44,64}$, V. Verzi ${ }^{25}$, G.P. Vettolani ${ }^{90}$, C. Veyssiere ${ }^{144}$, I. Viale ${ }^{55}$, A. Viana ${ }^{80}$, N. Viaux ${ }^{35}$, J. Vicha ${ }^{33}$, J. Vignatti ${ }^{35}$, C.F. Vigorito ${ }^{64,108}$, J. Villanueva ${ }^{98}$, J. Vink ${ }^{174}$, V. Vitale ${ }^{23}$, V. Vittorini ${ }^{99}$, V. Vodeb ${ }^{68}$, H. Voelk ${ }^{53}$, N. Vogel ${ }^{142}$, V. Voisin ${ }^{79}$, S. Vorobiov ${ }^{68}$, I. Vovk ${ }^{2}$, M. Vrastil ${ }^{33}$, T. Vuillaume ${ }^{45}$, S.J. Wagner ${ }^{170}$, R. Wagner ${ }^{105}$, P. Wagner ${ }^{52}$, K. Wakazono ${ }^{139}$, S.P. Wakely ${ }^{127}$,

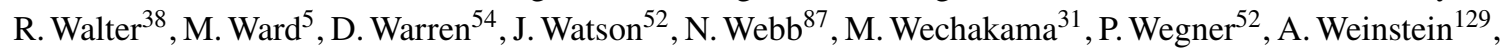
C. Weniger ${ }^{174}$, F. Werner ${ }^{53}$, H. Wetteskind ${ }^{105}$, M. White ${ }^{118}$, R. White ${ }^{53}$, A. Wierzcholska ${ }^{101}$, S. Wiesand ${ }^{52}$, R. Wijers ${ }^{174}$, M. Wilkinson ${ }^{124}$, M. Will ${ }^{105}$, D.A. Williams ${ }^{143}$, J. Williams ${ }^{124}$, T. Williamson ${ }^{162}$, A. Wolter ${ }^{95}$, Y.W. Wong ${ }^{142}$, M. Wood $^{96}$, C. Wunderlich ${ }^{62}$, T. Yamamoto ${ }^{213}$, H. Yamamoto $^{141}$, Y. Yamane ${ }^{141}$, R. Yamazaki ${ }^{209}$, S. Yanagita ${ }^{176}$, L. Yang ${ }^{205}$, S. Yoo ${ }^{180}$, T. Yoshida ${ }^{176}$, T. Yoshikoshi ${ }^{2}$, P. Yu ${ }^{71}$, P. Yu ${ }^{85}$, A. Yusafzai ${ }^{59}$, M.Zacharias ${ }^{20}$, G. Zaharijas $^{68}$, B.ZZaldivar ${ }^{14}$, L. Zampieri ${ }^{76}$, R. Zanmar Sanchez ${ }^{29}$, D.Zaric ${ }^{150}$, M. Zavrtanik ${ }^{68}$, D. Zavrtanik ${ }^{68}$, A.A. Zdziarski ${ }^{49}$, A. Zech ${ }^{20}$, H. Zechlin ${ }^{64}$, A. Zenin ${ }^{139}$, A. Zerwekh ${ }^{35}$, V.I. Zhdanov ${ }^{136}$, K. Ziętara ${ }^{169}$, A. Zink ${ }^{142}$, J. Ziółkowski ${ }^{49}$, V. Zitelli2 ${ }^{21}$, M. Živec ${ }^{68}$, A. Zmija ${ }^{142}$

1: Centre for Space Research, North-West University, Potchefstroom, 2520, South Africa 
2: Institute for Cosmic Ray Research, University of Tokyo, 5-1-5, Kashiwa-no-ha, Kashiwa, Chiba 277-8582, Japan

3 : Pontificia Universidad Católica de Chile, Av. Libertador Bernardo O’Higgins 340, Santiago, Chile

4 : AIM, CEA, CNRS, Université Paris-Saclay, Université Paris Diderot, Sorbonne Paris Cité, CEA ParisSaclay, IRFU/DAp, Bat 709, Orme des Merisiers, 91191 Gif-sur-Yvette, France

5 : Centre for Advanced Instrumentation, Dept. of Physics, Durham University, South Road, Durham DH1 3LE, United Kingdom

6 : Port d'Informació Científica, Edifici D, Carrer de l'Albareda, 08193 Bellaterrra (Cerdanyola del Vallès), Spain

7 : School of Physics and Astronomy, Monash University, Melbourne, Victoria 3800, Australia

8: Laboratoire Leprince-Ringuet, École Polytechnique (UMR 7638, CNRS/IN2P3, Institut Polytechnique de Paris), 91128 Palaiseau, France

9 : Department of Physics, Columbia University, 538 West 120th Street, New York, NY 10027, USA

10 : University of Oslo, Department of Physics, Sem Saelandsvei 24 - PO Box 1048 Blindern, N-0316 Oslo, Norway

11 : EMFTEL department and IPARCOS, Universidad Complutense de Madrid, 28040 Madrid, Spain

12 : Instituto de Astrofísica de Andalucía-CSIC, Glorieta de la Astronomía s/n, 18008, Granada, Spain

13 : Institute of Space Sciences (ICE-CSIC), and Institut d'Estudis Espacials de Catalunya (IEEC), and Institució Catalana de Recerca I Estudis Avançats (ICREA), Campus UAB, Carrer de Can Magrans, s/n 08193 Cerdanyola del Vallés, Spain

14 : Instituto de Física Teórica UAM/CSIC and Departamento de Física Teórica, Universidad Autónoma de Madrid, c/ Nicolás Cabrera 13-15, Campus de Cantoblanco UAM, 28049 Madrid, Spain

15 : Dublin Institute for Advanced Studies, 31 Fitzwilliam Place, Dublin 2, Ireland

16: Universidad Nacional Autónoma de México, Delegación Coyoacán, 04510 Ciudad de México, Mexico

17 : University of Geneva - Département de physique nucléaire et corpusculaire, 24 rue du Général-Dufour, 1211 Genève 4, Switzerland

18 : INFN Dipartimento di Scienze Fisiche e Chimiche - Università degli Studi dell'Aquila and Gran Sasso Science Institute, Via Vetoio 1, Viale Crispi 7, 67100 L'Aquila, Italy

19 : Instituto de Astronomia, Geofísico, e Ciências Atmosféricas - Universidade de São Paulo, Cidade Universitária, R. do Matão, 1226, CEP 05508-090, São Paulo, SP, Brazil

20 : LUTH, GEPI and LERMA, Observatoire de Paris, CNRS, PSL University, 5 place Jules Janssen, 92190, Meudon, France

21 : INAF - Osservatorio di Astrofisica e Scienza dello spazio di Bologna, Via Piero Gobetti 93/3, 40129 Bologna, Italy

22 : INAF - Osservatorio Astrofisico di Arcetri, Largo E. Fermi, 5 - 50125 Firenze, Italy

23 : INFN Sezione di Perugia and Università degli Studi di Perugia, Via A. Pascoli, 06123 Perugia, Italy

24 : INFN Sezione di Napoli, Via Cintia, ed. G, 80126 Napoli, Italy

25 : INFN Sezione di Roma Tor Vergata, Via della Ricerca Scientifica 1, 00133 Rome, Italy

26 : Argonne National Laboratory, 9700 S. Cass Avenue, Argonne, IL 60439, USA

27 : Aix-Marseille Université, CNRS/IN2P3, CPPM, 163 Avenue de Luminy, 13288 Marseille cedex 09, France

28 : INAF - Osservatorio Astronomico di Roma, Via di Frascati 33, 00040, Monteporzio Catone, Italy

29 : INAF - Osservatorio Astrofisico di Catania, Via S. Sofia, 78, 95123 Catania, Italy

30 : Grupo de Electronica, Universidad Complutense de Madrid, Av. Complutense s/n, 28040 Madrid, Spain

31 : National Astronomical Research Institute of Thailand, 191 Huay Kaew Rd., Suthep, Muang, Chiang Mai, 50200, Thailand

32 : Instituto de Astrofísica de Canarias and Departamento de Astrofísica, Universidad de La Laguna, La Laguna, Tenerife, Spain 
33 : FZU - Institute of Physics of the Czech Academy of Sciences, Na Slovance 1999/2, 18221 Praha 8, Czech Republic

34 : Astronomical Institute of the Czech Academy of Sciences, Bocni II 1401 - 14100 Prague, Czech Republic 35 : CCTVal, Universidad Técnica Federico Santa María, Avenida España 1680, Valparaíso, Chile

36 : ETH Zurich, Institute for Particle Physics, Schafmattstr. 20, CH-8093 Zurich, Switzerland

37 : The University of Manitoba, Dept of Physics and Astronomy, Winnipeg, Manitoba R3T 2N2, Canada

38 : Department of Astronomy, University of Geneva, Chemin d'Ecogia 16, CH-1290 Versoix, Switzerland

39: Laboratoire Univers et Particules de Montpellier, Université de Montpellier, CNRS/IN2P3, CC 72, Place Eugène Bataillon, F-34095 Montpellier Cedex 5, France

40 : Centro Brasileiro de Pesquisas Físicas, Rua Xavier Sigaud 150, RJ 22290-180, Rio de Janeiro, Brazil

41 : Institut de Fisica d'Altes Energies (IFAE), The Barcelona Institute of Science and Technology, Campus UAB, 08193 Bellaterra (Barcelona), Spain

42 : University of Groningen, KVI - Center for Advanced Radiation Technology, Zernikelaan 25, 9747 AA Groningen, The Netherlands

43 : School of Physics, University of New South Wales, Sydney NSW 2052, Australia

44 : INAF - Osservatorio Astrofisico di Torino, Strada Osservatorio 20, 10025 Pino Torinese (TO), Italy

45 : Univ. Savoie Mont Blanc, CNRS, Laboratoire d'Annecy de Physique des Particules - IN2P3, 74000 Annecy, France

46 : Department of Physics, TU Dortmund University, Otto-Hahn-Str. 4, 44221 Dortmund, Germany

47 : University of Zagreb, Faculty of electrical engineering and computing, Unska 3, 10000 Zagreb, Croatia

48 : University of Namibia, Department of Physics, 340 Mandume Ndemufayo Ave., Pioneerspark, Windhoek, Namibia

49 : Nicolaus Copernicus Astronomical Center, Polish Academy of Sciences, ul. Bartycka 18, 00-716 Warsaw, Poland

50: Universität Hamburg, Institut für Experimentalphysik, Luruper Chaussee 149, 22761 Hamburg, Germany 51 : Graduate School of Science, University of Tokyo, 7-3-1 Hongo, Bunkyo-ku, Tokyo 113-0033, Japan

52 : Deutsches Elektronen-Synchrotron, Platanenallee 6, 15738 Zeuthen, Germany

53 : Max-Planck-Institut für Kernphysik, Saupfercheckweg 1, 69117 Heidelberg, Germany

54 : RIKEN, Institute of Physical and Chemical Research, 2-1 Hirosawa, Wako, Saitama, 351-0198, Japan

55 : INFN Sezione di Padova and Università degli Studi di Padova, Via Marzolo 8, 35131 Padova, Italy

56 : Escuela Politécnica Superior de Jaén, Universidad de Jaén, Campus Las Lagunillas s/n, Edif. A3, 23071 Jaén, Spain

57 : Department of Physics and Electrical Engineering, Linnaeus University, 35195 Växjö, Sweden

58 : University of the Witwatersrand, 1 Jan Smuts Avenue, Braamfontein, 2000 Johannesburg, South Africa 59 : Institut für Theoretische Physik, Lehrstuhl IV: Plasma-Astroteilchenphysik, Ruhr-Universität Bochum, Universitätsstraße 150, 44801 Bochum, Germany

60 : Faculty of Physics and Applied Computer Science, University of Lódź, ul. Pomorska 149-153, 90-236 Lódź, Poland

61 : INAF - Istituto di Astrofisica Spaziale e Fisica Cosmica di Milano, Via A. Corti 12, 20133 Milano, Italy 62 : INFN and Università degli Studi di Siena, Dipartimento di Scienze Fisiche, della Terra e dell'Ambiente (DSFTA), Sezione di Fisica, Via Roma 56, 53100 Siena, Italy

63 : Center for Astrophysics | Harvard \& Smithsonian, 60 Garden St, Cambridge, MA 02180, USA

64 : INFN Sezione di Torino, Via P. Giuria 1, 10125 Torino, Italy

65 : Finnish Centre for Astronomy with ESO, University of Turku, Finland, FI-20014 University of Turku, Finland

66 : Pidstryhach Institute for Applied Problems in Mechanics and Mathematics NASU, 3B Naukova Street, Lviv, 79060, Ukraine

67 : Bhabha Atomic Research Centre, Trombay, Mumbai 400085, India 
68 : Center for Astrophysics and Cosmology, University of Nova Gorica, Vipavska 11c, 5270 Ajdovščina, Slovenia

69 : Institut für Astronomie und Astrophysik, Universität Tübingen, Sand 1, 72076 Tübingen, Germany

70 : Research School of Astronomy and Astrophysics, Australian National University, Canberra ACT 0200, Australia

71 : Department of Physics and Astronomy, University of California, Los Angeles, CA 90095, USA

72 : INFN Sezione di Bari and Politecnico di Bari, via Orabona 4, 70124 Bari, Italy

73 : Laboratoire de Physique des 2 infinis, Irene Joliot-Curie,IN2P3/CNRS, Université Paris-Saclay, Université de Paris, 15 rue Georges Clemenceau, 91406 Orsay, Cedex, France

74 : INFN Sezione di Pisa, Largo Pontecorvo 3, 56217 Pisa, Italy

75 : IRFU/DEDIP, CEA, Université Paris-Saclay, Bat 141, 91191 Gif-sur-Yvette, France

76 : INAF - Osservatorio Astronomico di Padova, Vicolo dell'Osservatorio 5, 35122 Padova, Italy

77 : INAF - Osservatorio Astronomico di Palermo "G.S. Vaiana", Piazza del Parlamento 1, 90134 Palermo, Italy

78 : School of Physics, University of Sydney, Sydney NSW 2006, Australia

79 : Sorbonne Université, Université Paris Diderot, Sorbonne Paris Cité, CNRS/IN2P3, Laboratoire de Physique Nucléaire et de Hautes Energies, LPNHE, 4 Place Jussieu, F-75005 Paris, France

80 : Instituto de Física de São Carlos, Universidade de São Paulo, Av. Trabalhador São-carlense, 400 - CEP 13566-590, São Carlos, SP, Brazil

81 : Departament de Física Quàntica i Astrofísica, Institut de Ciències del Cosmos, Universitat de Barcelona, IEEC-UB, Martí i Franquès, 1, 08028, Barcelona, Spain

82 : Department of Physics, Washington University, St. Louis, MO 63130, USA

83 : Saha Institute of Nuclear Physics, Bidhannagar, Kolkata-700 064, India

84 : INAF - Osservatorio Astronomico di Capodimonte, Via Salita Moiariello 16, 80131 Napoli, Italy

85 : Université de Paris, CNRS, Astroparticule et Cosmologie, 10, rue Alice Domon et Léonie Duquet, 75013 Paris Cedex 13, France

86 : Astronomy Department of Faculty of Physics, Sofia University, 5 James Bourchier Str., 1164 Sofia, Bulgaria

87 : Institut de Recherche en Astrophysique et Planétologie, CNRS-INSU, Université Paul Sabatier, 9 avenue Colonel Roche, BP 44346, 31028 Toulouse Cedex 4, France

88 : School of Physics and Astronomy, University of Minnesota, 116 Church Street S.E. Minneapolis, Minnesota 55455-0112, USA

89 : IRFU, CEA, Université Paris-Saclay, Bât 141, 91191 Gif-sur-Yvette, France

90 : INAF - Istituto di Radioastronomia, Via Gobetti 101, 40129 Bologna, Italy

91 : INAF - Istituto di Astrofisica Spaziale e Fisica Cosmica di Palermo, Via U. La Malfa 153, 90146

Palermo, Italy

92 : Astronomical Observatory, Department of Physics, University of Warsaw, Aleje Ujazdowskie 4, 00478 Warsaw, Poland

93 : Armagh Observatory and Planetarium, College Hill, Armagh BT61 9DG, United Kingdom

94 : INFN Sezione di Catania, Via S. Sofia 64, 95123 Catania, Italy

95 : INAF - Osservatorio Astronomico di Brera, Via Brera 28, 20121 Milano, Italy

96 : Kavli Institute for Particle Astrophysics and Cosmology, Department of Physics and SLAC National Accelerator Laboratory, Stanford University, 2575 Sand Hill Road, Menlo Park, CA 94025, USA

97 : Universidade Cruzeiro do Sul, Núcleo de Astrofísica Teórica (NAT/UCS), Rua Galvão Bueno 8687, Bloco B, sala 16, Libertade 01506-000 - São Paulo, Brazil

98 : Universidad de Valparaíso, Blanco 951, Valparaiso, Chile

99 : INAF - Istituto di Astrofisica e Planetologia Spaziali (IAPS), Via del Fosso del Cavaliere 100, 00133

Roma, Italy 
100 : Lund Observatory, Lund University, Box 43, SE-22100 Lund, Sweden

101 : The Henryk Niewodniczański Institute of Nuclear Physics, Polish Academy of Sciences, ul. Radzikowskiego 152, 31-342 Cracow, Poland

102 : Escola de Engenharia de Lorena, Universidade de São Paulo, Área I - Estrada Municipal do Campinho, $\mathrm{s} / \mathrm{n}^{\circ}, \mathrm{CEP}$ 12602-810, Pte. Nova, Lorena, Brazil

103 : INFN Sezione di Trieste and Università degli Studi di Udine, Via delle Scienze 208, 33100 Udine, Italy

104 : Palacky University Olomouc, Faculty of Science, RCPTM, 17. listopadu 1192/12, 77146 Olomouc,

Czech Republic

105 : Max-Planck-Institut für Physik, Föhringer Ring 6, 80805 München, Germany

106 : CENBG, Univ. Bordeaux, CNRS-IN2P3, UMR 5797, 19 Chemin du Solarium, CS 10120, F-33175

Gradignan Cedex, France

107 : Dublin City University, Glasnevin, Dublin 9, Ireland

108 : Dipartimento di Fisica - Universitá degli Studi di Torino, Via Pietro Giuria 1 - 10125 Torino, Italy

109 : Tata Institute of Fundamental Research, Homi Bhabha Road, Colaba, Mumbai 400005, India

110 : Universitá degli Studi di Napoli "Federico II" - Dipartimento di Fisica "E. Pancini", Complesso universitario di Monte Sant'Angelo, Via Cintia - 80126 Napoli, Italy

111 : Oskar Klein Centre, Department of Physics, University of Stockholm, Albanova, SE-10691, Sweden

112 : Yale University, Department of Physics and Astronomy, 260 Whitney Avenue, New Haven, CT 06520-8101, USA

113 : CIEMAT, Avda. Complutense 40, 28040 Madrid, Spain

114 : University of Oxford, Department of Physics, Denys Wilkinson Building, Keble Road, Oxford OX1 3RH, United Kingdom

115 : School of Physics \& Astronomy, University of Southampton, University Road, Southampton SO17

1BJ, United Kingdom

116 : Department of Physics and Technology, University of Bergen, Museplass 1, 5007 Bergen, Norway

117 : Western Sydney University, Locked Bag 1797, Penrith, NSW 2751, Australia

118 : School of Physical Sciences, University of Adelaide, Adelaide SA 5005, Australia

119 : INFN Sezione di Roma La Sapienza, P.le Aldo Moro, 2 - 00185 Roma, Italy

120 : INFN Sezione di Bari, via Orabona 4, 70126 Bari, Italy

121 : University of Rijeka, Department of Physics, Radmile Matejcic 2, 51000 Rijeka, Croatia

122 : Institute for Theoretical Physics and Astrophysics, Universität Würzburg, Campus Hubland Nord, Emil-Fischer-Str. 31, 97074 Würzburg, Germany

123 : Universidade Federal Do Paraná - Setor Palotina, Departamento de Engenharias e Exatas, Rua Pioneiro, 2153, Jardim Dallas, CEP: 85950-000 Palotina, Paraná, Brazil

124 : Dept. of Physics and Astronomy, University of Leicester, Leicester, LE1 7RH, United Kingdom

125 : Univ. Grenoble Alpes, CNRS, IPAG, 414 rue de la Piscine, Domaine Universitaire, 38041 Grenoble Cedex 9, France

126 : National Centre for nuclear research (Narodowe Centrum Badań Jądrowych), U1. Andrzeja Sołtana7, 05-400 Otwock, Świerk, Poland

127 : Enrico Fermi Institute, University of Chicago, 5640 South Ellis Avenue, Chicago, IL 60637, USA

128 : Institut für Physik \& Astronomie, Universität Potsdam, Karl-Liebknecht-Strasse 24/25, 14476 Potsdam, Germany

129 : Department of Physics and Astronomy, Iowa State University, Zaffarano Hall, Ames, IA 50011-3160, USA

130 : School of Physics, Aristotle University, Thessaloniki, 54124 Thessaloniki, Greece

131 : King's College London, Strand, London, WC2R 2LS, United Kingdom

132 : Escola de Artes, Ciências e Humanidades, Universidade de São Paulo, Rua Arlindo Bettio, CEP 03828-000, 1000 São Paulo, Brazil 
133 : Dept. of Astronomy \& Astrophysics, Pennsylvania State University, University Park, PA 16802, USA

134 : National Technical University of Athens, Department of Physics, Zografos 9, 15780 Athens, Greece

135 : University of Wisconsin, Madison, 500 Lincoln Drive, Madison, WI, 53706, USA

136 : Astronomical Observatory of Taras Shevchenko National University of Kyiv, 3 Observatorna Street, Kyiv, 04053, Ukraine

137 : Department of Physics, Purdue University, West Lafayette, IN 47907, USA

138 : Unitat de Física de les Radiacions, Departament de Física, and CERES-IEEC, Universitat Autònoma de Barcelona, Edifici C3, Campus UAB, 08193 Bellaterra, Spain

139 : Institute for Space-Earth Environmental Research, Nagoya University, Chikusa-ku, Nagoya 464-8601, Japan

140 : Department of Physical Science, Hiroshima University, Higashi-Hiroshima, Hiroshima 739-8526, Japan

141 : Department of Physics, Nagoya University, Chikusa-ku, Nagoya, 464-8602, Japan

142 : Friedrich-Alexander-Universität Erlangen-Nürnberg, Erlangen Centre for Astroparticle Physics (ECAP), Erwin-Rommel-Str. 1, 91058 Erlangen, Germany

143 : Santa Cruz Institute for Particle Physics and Department of Physics, University of California, Santa Cruz, 1156 High Street, Santa Cruz, CA 95064, USA

144 : IRFU / DIS, CEA, Université de Paris-Saclay, Bat 123, 91191 Gif-sur-Yvette, France

145 : INFN Sezione di Trieste and Università degli Studi di Trieste, Via Valerio 2 I, 34127 Trieste, Italy

146 : School of Physics \& Center for Relativistic Astrophysics, Georgia Institute of Technology, 837 State Street, Atlanta, Georgia, 30332-0430, USA

147 : Alikhanyan National Science Laboratory, Yerevan Physics Institute, 2 Alikhanyan Brothers St., 0036, Yerevan, Armenia

148 : INAF - Telescopio Nazionale Galileo, Roche de los Muchachos Astronomical Observatory, 38787 Garafia, TF, Italy

149 : INFN Sezione di Bari and Università degli Studi di Bari, via Orabona 4, 70124 Bari, Italy

150 : University of Split - FESB, R. Boskovica 32, 21000 Split, Croatia

151: Universidad Andres Bello, República 252, Santiago, Chile

152 : Academic Computer Centre CYFRONET AGH, ul. Nawojki 11, 30-950 Cracow, Poland

153 : University of Liverpool, Oliver Lodge Laboratory, Liverpool L69 7ZE, United Kingdom

154 : Department of Physics, Yamagata University, Yamagata, Yamagata 990-8560, Japan

155 : Astronomy Department, Adler Planetarium and Astronomy Museum, Chicago, IL 60605, USA

156 : Faculty of Management Information, Yamanashi-Gakuin University, Kofu, Yamanashi 400-8575, Japan

157 : Department of Physics, Tokai University, 4-1-1, Kita-Kaname, Hiratsuka, Kanagawa 259-1292, Japan

158 : Centre for Astrophysics Research, Science \& Technology Research Institute, University of Hertfordshire, College Lane, Hertfordshire AL10 9AB, United Kingdom

159 : Cherenkov Telescope Array Observatory, Saupfercheckweg 1, 69117 Heidelberg, Germany

160 : Tohoku University, Astronomical Institute, Aobaku, Sendai 980-8578, Japan

161 : Department of Physics, Rikkyo University, 3-34-1 Nishi-Ikebukuro, Toshima-ku, Tokyo, Japan

162 : Department of Physics and Astronomy and the Bartol Research Institute, University of Delaware, Newark, DE 19716, USA

163 : Institut für Astro- und Teilchenphysik, Leopold-Franzens-Universität, Technikerstr. 25/8, 6020 Innsbruck, Austria

164 : Department of Physics and Astronomy, University of Utah, Salt Lake City, UT 84112-0830, USA

165 : IMAPP, Radboud University Nijmegen, P.O. Box 9010, 6500 GL Nijmegen, The Netherlands

166 : Josip Juraj Strossmayer University of Osijek, Trg Ljudevita Gaja 6, 31000 Osijek, Croatia

167 : Department of Earth and Space Science, Graduate School of Science, Osaka University, Toyonaka 560-0043, Japan 
168 : Yukawa Institute for Theoretical Physics, Kyoto University, Kyoto 606-8502, Japan

169 : Astronomical Observatory, Jagiellonian University, ul. Orla 171, 30-244 Cracow, Poland

170 : Landessternwarte, Zentrum für Astronomie der Universität Heidelberg, Königstuhl 12, 69117 Heidel-

berg, Germany

171 : University of Alabama, Tuscaloosa, Department of Physics and Astronomy, Gallalee Hall, Box 870324 Tuscaloosa, AL 35487-0324, USA

172 : Department of Physics, University of Bath, Claverton Down, Bath BA2 7AY, United Kingdom

173 : University of Iowa, Department of Physics and Astronomy, Van Allen Hall, Iowa City, IA 52242, USA

174 : Anton Pannekoek Institute/GRAPPA, University of Amsterdam, Science Park 9041098 XH Amsterdam, The Netherlands

175 : Faculty of Computer Science, Electronics and Telecommunications, AGH University of Science and Technology, Kraków, al. Mickiewicza 30, 30-059 Cracow, Poland

176 : Faculty of Science, Ibaraki University, Mito, Ibaraki, 310-8512, Japan

177 : Faculty of Science and Engineering, Waseda University, Shinjuku, Tokyo 169-8555, Japan

178 : Institute of Astronomy, Faculty of Physics, Astronomy and Informatics, Nicolaus Copernicus University in Toruń, ul. Grudziądzka 5, 87-100 Toruń, Poland

179 : Graduate School of Science and Engineering, Saitama University, 255 Simo-Ohkubo, Sakura-ku, Saitama city, Saitama 338-8570, Japan

180 : Division of Physics and Astronomy, Graduate School of Science, Kyoto University, Sakyo-ku, Kyoto, 606-8502, Japan

181 : Centre for Quantum Technologies, National University Singapore, Block S15, 3 Science Drive 2, Singapore 117543, Singapore

182 : Institute of Particle and Nuclear Studies, KEK (High Energy Accelerator Research Organization), 1-1

Oho, Tsukuba, 305-0801, Japan

183 : Department of Physics and Astronomy, University of Sheffield, Hounsfield Road, Sheffield S3 7RH, United Kingdom

184 : Centro de Ciências Naturais e Humanas, Universidade Federal do ABC, Av. dos Estados, 5001, CEP: 09.210-580, Santo André - SP, Brazil

185 : Dipartimento di Fisica e Astronomia, Sezione Astrofisica, Universitá di Catania, Via S. Sofia 78, I-95123 Catania, Italy

186 : Department of Physics, Humboldt University Berlin, Newtonstr. 15, 12489 Berlin, Germany

187 : Texas Tech University, 2500 Broadway, Lubbock, Texas 79409-1035, USA

188 : University of Zielona Góra, ul. Licealna 9, 65-417 Zielona Góra, Poland

189 : Institute for Nuclear Research and Nuclear Energy, Bulgarian Academy of Sciences, 72 boul. Tsarigradsko chaussee, 1784 Sofia, Bulgaria

190 : University of Białystok, Faculty of Physics, ul. K. Ciołkowskiego 1L, 15-254 Białystok, Poland

191 : Faculty of Physics, National and Kapodestrian University of Athens, Panepistimiopolis, 15771 Ilissia, Athens, Greece

192 : Universidad de Chile, Av. Libertador Bernardo O’Higgins 1058, Santiago, Chile

193 : Hiroshima Astrophysical Science Center, Hiroshima University, Higashi-Hiroshima, Hiroshima 7398526, Japan

194 : Department of Applied Physics, University of Miyazaki, 1-1 Gakuen Kibana-dai Nishi, Miyazaki, 889-2192, Japan

195 : School of Allied Health Sciences, Kitasato University, Sagamihara, Kanagawa 228-8555, Japan

196 : Departamento de Astronomía, Universidad de Concepción, Barrio Universitario S/N, Concepción, Chile

197 : Charles University, Institute of Particle \& Nuclear Physics, V Holešovičkách 2, 18000 Prague 8, Czech Republic 
198 : Astronomical Observatory of Ivan Franko National University of Lviv, 8 Kyryla i Mephodia Street, Lviv, 79005, Ukraine

199 : Kobayashi-Maskawa Institute (KMI) for the Origin of Particles and the Universe, Nagoya University, Chikusa-ku, Nagoya 464-8602, Japan

200 : Graduate School of Technology, Industrial and Social Sciences, Tokushima University, Tokushima 770-8506, Japan

201 : Space Research Centre, Polish Academy of Sciences, ul. Bartycka 18A, 00-716 Warsaw, Poland

202 : Instituto de Física - Universidade de São Paulo, Rua do Matão Travessa R Nr.187 CEP 05508-090 Cidade Universitária, São Paulo, Brazil

203 : International Institute of Physics at the Federal University of Rio Grande do Norte, Campus Universitário, Lagoa Nova CEP 59078-970 Rio Grande do Norte, Brazil

204 : University College Dublin, Belfield, Dublin 4, Ireland

205 : Centre for Astro-Particle Physics (CAPP) and Department of Physics, University of Johannesburg, PO Box 524, Auckland Park 2006, South Africa

206 : Departamento de Física, Facultad de Ciencias Básicas, Universidad Metropolitana de Ciencias de la Educación, Santiago, Chile

207 : Núcleo de Formação de Professores - Universidade Federal de São Carlos, Rodovia Washington Luís, km 235 CEP 13565-905 - SP-310 São Carlos - São Paulo, Brazil

208 : Physik-Institut, Universität Zürich, Winterthurerstrasse 190, 8057 Zürich, Switzerland

209 : Department of Physical Sciences, Aoyama Gakuin University, Fuchinobe, Sagamihara, Kanagawa, 252-5258, Japan

210 : University of the Free State, Nelson Mandela Avenue, Bloemfontein, 9300, South Africa

211 : Faculty of Electronics and Information, Warsaw University of Technology, ul. Nowowiejska 15/19, 00-665 Warsaw, Poland

212 : Rudjer Boskovic Institute, Bijenicka 54, 10000 Zagreb, Croatia

213 : Department of Physics, Konan University, Kobe, Hyogo, 658-8501, Japan

214 : Kumamoto University, 2-39-1 Kurokami, Kumamoto, 860-8555, Japan

215 : University School for Advanced Studies IUSS Pavia, Palazzo del Broletto, Piazza della Vittoria 15, 27100 Pavia, Italy

216 : Aalto University, Otakaari 1, 00076 Aalto, Finland

217 : Agenzia Spaziale Italiana (ASI), 00133 Roma, Italy

218 : Observatoire de la Cote d'Azur, Boulevard de l'Observatoire CS34229, 06304 Nice Cedex 4, Franc 